\title{
The Mumford-Tate Conjecture for Drinfeld-Modules
}

By

\author{
Richard PINK*
}

\begin{abstract}
Consider the Galois representation on the Tate module of a Drinfeld module over a finitely generated field in generic characteristic. The main object of this paper is to determine the image of Galois in this representation, up to commensurability. We also determine the Dirichlet density of the set of places of prescribed reduction type, such as places of ordinary reduction.
\end{abstract}

\section{§0. Introduction}

Let $F$ be a finitely generated field of transcendence degree 1 over a finite field of characteristic $p$. Fix a place $\infty$ of $F$, and let $A$ be the ring of elements of $F$ which are regular outside $\infty$. Consider a finitely generated extension $K$ of $F$ and a Drinfeld module $\varphi: A \rightarrow \operatorname{End}_{K}\left(\boldsymbol{G}_{\boldsymbol{a}}\right)$ of rank $n \geq 1$ (cf. Drinfeld [10]). In other words $K$ is a finitely generated field of transcendece degree $\geq 1$ over $\boldsymbol{F}_{p}$, and $\varphi$ has "generic characteristic". Let $K^{\text {sep }} \subset \bar{K}$ denote a separable, respectively algebraic closure of $K$. Let $F_{\lambda}$ denote the completion of $F$ at a place $\lambda$. If $\lambda \neq \infty$ we have a continuous representation

$$
\rho_{\lambda}: \operatorname{Gal}\left(K^{\mathrm{sep}} / K\right) \rightarrow \mathrm{GL}_{n}\left(F_{\lambda}\right)
$$

which describes the Galois action on the $\lambda$-adic Tate module of $\varphi$. The main goal of this article is to give a qualitative characterization of the image of $\rho_{\lambda}$. Here the term "qualitative" refers to properties that are shared by all open subgroups, i.e. to those properties that do not change under replacing $K$ by a finite extension. Our method actually applies to any given finite number of places simultaneously and shows that the image of Galois is as

Communicated by Y. Ihara, September 20, 1996.

1991 Mathematics Subject Classifications: 11G09, 11R58, 11R45

* Fakultät für Mathematik und Informatik, Universität Mannheim, D-68131 Mannheim, Germany. 
big as possible.

Theorem 0.耳. Suppose that $\operatorname{End}_{\bar{K}}(\varphi)=A$. Then for any finite set $\Lambda$ of places $\lambda \neq \infty$ of $F$ the image of the homomorphism

$$
\operatorname{Gal}\left(K^{\mathrm{sep}} / K\right) \rightarrow \prod_{\lambda \in \Lambda} \mathrm{GL}_{n}\left(F_{\lambda}\right)
$$

is open.

More generally, the endomorphism ring $\operatorname{End}_{K}(\varphi)$ acts on the Tate module and commutes with the $\lambda$-adic representation. In other words, the image of Galois lies in the centralizer $\operatorname{Cent}_{\mathrm{GL}_{n}\left(F_{\lambda}\right)}\left(\operatorname{End}_{K}(\varphi)\right)$. After replacing $K$ by a finite extension we may assume that all endomorphisms of $\varphi$ over an algebraic closure of $K$ are already defined over $K$.

Theorem 0.2. Suppose that $\operatorname{End}_{\bar{K}}(\varphi)=\operatorname{End}_{K}(\varphi)$. Then for any finite set $\Lambda$ of places $\lambda \neq \infty$ of $F$ the image of the homomorphism

$$
\operatorname{Gal}\left(K^{\mathrm{sep}} / K\right) \rightarrow \prod_{\lambda \in \Lambda} \operatorname{Cent}_{\mathrm{GL}_{n}\left(F_{\lambda}\right)}\left(\operatorname{End}_{K}(\varphi)\right)
$$

is open.

It would be interesting to extend these results to the set of all finite places of $F$, i.e. to determine the image of Galois in the adelic representation. But this will require additional techniques of a different nature. The author hopes to come back to this problem in the future.

Places of Prescribed Reduction Type. The proof of Theorems $0.1-2$ is modeled largely on Serre's analysis of the l-adic representations arising from abelian varieties (see [26], [28], [29], [30], resp. Chi [4]). One crucial ingredient is the study of Frobenius elements associated to the reductions of $\varphi$ over finite fields, and in particular of their Newton polygons. The problem is thus connected with the question of how often a given Newton polygon occurs. In the analogous case of low dimensional abelian varieties, for instance for elliptic curves without potential complex multiplication, it is known that the set of primes of ordinary reduction has Dirichlet density 1 (cf. Serre [26], Ogus [24] Prop. 2.7). The following result is an analogue of that 
fact. Let $X$ be an integral scheme of finite type over $F_{p}$ whose function field is $K$.

Theorem 0.3. Let $\Delta$ be the finite quotient of $\mathrm{Gal}\left(K^{\mathrm{sep}} / K\right)$ which acts faithfully on $\operatorname{End}_{\bar{K}}(\varphi)$. Let $p^{e}$ be the degree of the totally inseparable part of $\operatorname{End}_{\bar{K}}(\varphi)$ over $A$.

(a) For any closed point $x \in X$ where $\varphi$ has good reduction the height of this reduction is divisible by $p^{e}$.

(b) For any integer $l \geq 1$ the set of closed points $x \in X$ at which $\varphi$ has good reduction of height $p^{e} l$ has Dirichlet density

$$
\frac{\operatorname{card}\{\delta \in \Delta \mid \operatorname{ord}(\delta)=l\}}{\operatorname{card}(\Delta)}
$$

In particular, note the following special case of Theorem 0.3.

Corollary 0.4. Suppose that $\operatorname{End}_{\bar{K}}(\varphi)=A$. Then the set of closed points $x \in X$ where $\varphi$ has good ordinary reduction has Dirichlet density 1.

If a Newton polygon is not forbidden by part (a) of Theorem 0.3 , but its occurrences have Dirichlet density 0 , it is natural to ask whether there are nevertheless infinitely many reductions with this Newton polygon and how sparsely they are distributed. The methods of this article do not illuminate this problem. (However, for some recent results in this direction see Brown [3], David [5].)

Motivation. The title of this article calls for a few explanations. It is based on the principle that Drinfeld modules play the same role for function fields that abelian varieties play for number fields. Consider an abelian variety $A$ of dimension $d$ over a number field $K$. Embed $K$ into the complex numbers and consider the singular homology group $V:=H_{1}(A(C), Q)$. This is a $Q$-vector space of dimension $2 d$ possessing a natural Hodge structure of type $\{(0,-1),(-1,0)\}$. That is, its Hodge filtration is a descending filtration of $V_{\boldsymbol{C}}:=V \otimes_{\mathbf{Q}} C$ by $C$-vector spaces $\mathrm{Fil}^{i} V_{\boldsymbol{C}}$ satisfying $\mathrm{Fil}^{-1} V_{\boldsymbol{C}}=\mathrm{Fil}^{0} V_{\boldsymbol{C}} \oplus \overline{\mathrm{Fil}^{0} V_{\boldsymbol{C}}}=V_{\boldsymbol{C}}$ and $\mathrm{Fil}^{1} V_{C}=0$. For any $d \geq 0$ the tensor space $T^{d, d}:=V^{\otimes d} \otimes\left(V^{\vee}\right)^{\otimes d}$ also inherits a Hodge structure, i.e. a filtration $\mathrm{Fil}^{\circ} T_{\boldsymbol{C}}^{d . d}$ with certain properties. The elements of $T^{d, d} \cap \mathrm{Fil}^{0} T_{C}^{d, d}$ are called Hodge cycles of the original Hodge structure. Choosing an identification $V \cong Q^{2 d}$ the Hodge group (or MumfordTate group) is defined as the subgroup $G \subset \mathrm{GL}_{2 d, \boldsymbol{Q}}$ fixing all Hodge cycles 
for all $d$. On the other hand the rational $l$-adic Tate module of $A$ is naturally isomorphic to $V \otimes_{Q} Q_{l}$ and carries a continuous action of $\operatorname{Gal}(\bar{K} / K)$. The image of this representation is a compact subgroup $\Gamma_{l} \subset \mathrm{GL}_{2 d}\left(\boldsymbol{Q}_{l}\right)$, and the Mumford-Tate conjecture states that some open subgroup of $\Gamma_{l}$ is open in $G\left(Q_{l}\right)$. In fact, this assertion is a consequence of certain general (unproved) principles for motives and motivic Galois groups, which will not be explained here. Certain parts of the Mumford-Tate conjecture have been proved: among others see Deligne et al. [8], Serre [26], [28], [29], [30], resp. Chi [4]. Note that the Mumford-Tate conjecture can be read in two ways. We shall take the point of view that it essentially determines the image of Galois when the Hodge group is known.

Let us work out the analogies in the Drinfeld module case. Let $\varphi$ etc. be as above. Let $C_{\infty}$ denote the completion of the algebraic closure of $F_{\infty}$ and extend the embedding $F \subset C_{\infty}$ to $K \subseteq C_{\infty}$. Then $\varphi$ possesses an "analytic uniformization" by a projective $A$-submodule $M \subset C_{\infty}$ of finite type and rank $n$ (see, e.g., Drinfeld [10] §3). Put $V:=M \otimes_{A} F$ and let $\mathrm{Fil}^{0} V_{C_{\infty}}$ denote the kernel of the canonical homomorphism $V_{\boldsymbol{C}_{\infty}}:=V \otimes_{F} C_{\infty} \rightarrow C_{\infty}, v \otimes x \mapsto v x$. This is a $C_{\infty}$-subspace of codimension 1 , which contains no non-zero element of $V \otimes_{F} F_{\infty}$. (For the relation with de Rham cohomology see Gekeler [14].) Set $\mathrm{Fil}^{-1} V_{\boldsymbol{C}_{\infty}}:=V_{\boldsymbol{C}_{\infty}}$ and $\mathrm{Fil}^{1} V_{\boldsymbol{C}_{\infty}}:=0$. By general principles (cf. Anderson [1]) a Drinfeld module can be viewed as a "pure motive of rank $n$ and weight $1 / n$ ", so we interpret this filtration as a pure Hodge structure of weight $1 / n$ on $V$.

To this object there should be associated a "Hodge group" $G$, which is an algebraic subgroup of $\mathrm{GL}_{n, F}$ once a basis of $V$ has been chosen. It is tempting to define it as the stabilizer of all "Hodge cycles", in the same way as above. Whatever the correct definition may be, it is natural to expect

Guess 0.5. $G=\operatorname{Cent}_{\mathrm{GL}_{n, F}}\left(\operatorname{End}_{\bar{K}}(\varphi)\right)$.

Namely, for any $\lambda \neq \infty$ the rational $\lambda$-adic Tate module of $\varphi$ is canonically isomorphic to $V \otimes_{F} F_{\lambda}$. Thus the image of Galois can be compared with $G$ and, assuming Guess 0.5 , Theorem 0.2 states that the image of Galois is open in the Hodge group, just as in the abelian variety case. To remain in keeping with general principles, Guess 0.5 should be proved by purely algebraic means, using only the information on the Hodge filtration that was stated above. Also, one should give a conceptual proof within the framework of a general theory of such Hodge structures. Since all this would go beyond the scope of this paper, we refrain from discussing these matters further. 
Outline of the Article. The proof of Theorem 0.2 follows roughly the lines laid out by the above motivational remarks. The general case can be reduced to that of Theorem 0.1 , so we may assume $\operatorname{End}_{\bar{K}}(\varphi)=A$. Let $G_{\lambda} \subset \mathrm{GL}_{n, F_{\lambda}}$ denote the Zariski closure of the image of $\rho_{\lambda}$. By recent results of Taguchi, respectively Tamagawa, comprising in particular the Tate conjecture for Drinfeld modules, the tautological representation of $G_{\lambda}^{\circ}$ is absolutely irreducible. Using this information, the existence of places of ordinary reduction, and some arguments from the representation theory of linear algebraic groups we can then deduce $G_{\lambda}=\mathrm{GL}_{n, F_{\lambda}}$.

Next, the question of openness has two parts, corresponding to the factorization $1 \rightarrow \mathrm{SL}_{n} \rightarrow \mathrm{GL}_{n} \stackrel{\text { det }}{\rightarrow} \boldsymbol{G}_{m} \rightarrow 1$. The image of Galois under the determinant map is characterized by results of Hayes concerning the abelian class field theory of $F$. For the semisimple part we are led to the purely group theoretical problem of studying Zariski dense compact subgroups of $\mathrm{SL}_{n}$ and $\mathrm{PGL}_{n}$ over the completion of $F$ at one or a finite number of places. If we had $F=Q$ it would be well-known and easy to show that such a subgroup is open. But here the function field case is significantly more involved. A detailed analysis of such subgroups has - in greater generality - been carried out by this author in the separate article [25]. The main result of that paper, combined with some additional arithmetic information about $\varphi$, implies the desired openness.

An effort has been made to present uniform proofs for all fields $K$ that are finitely generated over $F$. It is hoped that the reader will find some advantages in this principle. In fact, the arguments hardly simplify when $K$ is assumed to be finite over $F$. Only in Theorem 1.4 (Taguchi's semisimplicity theorem) it was necessary to obtain the general result by reduction to this case.

The rest of this article is structured as follows. In $\S 1$ we fix notations and collect all known facts on Drinfeld modules that will be needed. $\$ 2$ contains the proof of Theorems $0.1-2$, modulo results from the appendices. In $\S 3$ we prove Theorem 0.3 . There are also two appendices which are independent of the rest of the article. Appendix A contains some results from the representation theory of linear algebraic groups which are used in $\S 2$. Finally, Appendix B discusses the concept of Dirichlet density for schemes of arbitrary dimension, for which no suitable reference was found.

Last, but not least, the author wishes to express his gratitude to the institutions and their members that made this work possible. The essential 
arguments were found during a stay at the Research Institute for Mathematical Sciences at Kyōto University in Spring 1994, that was supported partly by the Japan Association for Mathematical Science. The author extends special thanks to Takayuki Oda for inviting him to Japan and to Akio Tamagawa for giving the stimulus for this work and for many valuable discussions. Thanks are also due to the referee for pointing out a number of minor mistates.

\section{$\S 1$. Ingredients from the Arithmetic of Drinfeld Modules}

Throughout the article the notations and assumptions of the introduction remain in order. For the fundamentals on Drinfeld modules we refer to Drinfeld's original article [10], to Deligne-Husemoller [9], Goss [16] and [17], or Hayes [19].

The endomorphism ring. For any extension field $K^{\prime}$ of $K$ the endomorphism ring $\operatorname{End}_{K^{\prime}}(\varphi)$ consists of the elements of $\operatorname{End}_{K^{\prime}}\left(G_{a}\right)$ which commute with $\varphi(A)$. It is known that $\operatorname{End}_{K^{\prime}}(\varphi)$ has no zero-divisors and is projective of finite type as module over $A$. Since we are in generic characteristic, it is also commutative and of $A$-rank at most $n$ (see [10] $\S 2 \mathrm{C}$ ). In particular, this implies that all endomorphisms over $\bar{K}$ are defined already over a fixed finite extension of $K$.

Let us abbreviate $A^{\prime}:=\operatorname{End}_{\bar{\kappa}}(\varphi)$ and $F^{\prime}:=\operatorname{Quot}\left(A^{\prime}\right)$. Identifying $A$ with its image in $A^{\prime}$, the homomorphism $\varphi: A \rightarrow \operatorname{End}_{K}\left(G_{a}\right)$ extends to a (tautological) homomorphism $\varphi^{\prime}: A^{\prime} \rightarrow \operatorname{End}_{\bar{K}}\left(G_{a}\right)$. This is again a Drinfeld module, except that $A^{\prime}$ may be a non-maximal order in $F^{\prime}$. There are two ways of dealing with that phenomenon. Following Hayes [18] we could work with non-maximal orders throughout the article, with essentially no changes. Alternatively, we can modify $\varphi$ by a suitable isogeny. Let $\tilde{A}^{\prime}$ be the normalization of $A^{\prime}$ in $F^{\prime}$. By [18] Prop. 3.2 we have:

Proposition 1.1. There is a Drinfeld module $\psi^{\prime}: \tilde{A}^{\prime} \rightarrow \operatorname{End}_{\bar{K}}\left(G_{a}\right)$ such that $\left.\psi^{\prime}\right|_{A^{\prime}}$ is isogenous to $\varphi^{\prime}$, i.e. there exists a non-zero $f \in \operatorname{End}_{K}\left(\mathbb{G}_{a}\right)$ such that $f \circ \varphi^{\prime}(x)=\psi^{\prime}(x) \circ f$ for all $x \in A^{\prime}$. Moreover, $\psi^{\prime}$ can be chosen such that the restriction $\left.\psi^{\prime}\right|_{A}$ is defined over $K$.

Specialization. Since $A$ is a finitely generated ring, the coefficients of all elements in $\varphi(A) \subset \operatorname{End}_{K}\left(G_{a}\right)$ lie in a finitely generated subring $R \subset K$. After enlarging $R$ we may suppose that $K=\mathrm{Quot}(R)$. Moreover, after inverting 
finitely many elements the highest coefficients become units in $R$. Then $X:=\operatorname{Spec}(R)$ is a model of $K$ of finite type over $\operatorname{Spec}\left(F_{p}\right)$, and by construction $\varphi$ defines a family of Drinfeld modules of rank $n$ over $X$. In particular, for any point $x \in X$ we obtain a Drinfeld module $\varphi_{x}: A \rightarrow \operatorname{End}_{k_{x}}\left(G_{a}\right)$ of rank $n$ defined over the residue field $k_{x}$.

Let $d: \operatorname{End}_{R}\left(G_{a}\right) \rightarrow R$ denote the derivative at the origin of $G_{a}$, i.e. the action on the Lie algebra of $G_{a}$. Then $d \circ \varphi: A \rightarrow R$ corresponds to a natural morphism $X \rightarrow \operatorname{Spec} A=C \backslash\{\infty\}$, where $C$ is the smooth projective curve with function field $F$. The image point of $x \in X$ is denoted $\lambda_{x}$. We say that $\varphi_{x}$ has generic or special characteristic according to whether $\lambda_{x}$ is the generic or a closed point of $C$. For instance, $\varphi$ itself has generic characteristic. If $\lambda_{x}$ is a closed point of $C$, we identify it with the associated valuation on $F$.

The Tate-module. Consider any place $\lambda \neq \infty$ of $F$ and let $\mathfrak{p}_{\lambda} \subset A$ denote the corresponding maximal ideal. Let $k_{x}^{\text {sep }}$ denote a separable closure of the residue field $k_{x}$. For any integer $i \geq 0$ the elements of $k_{x}^{\text {sep }}$ annihilated by all the endomorphisms in $\varphi\left(\mathfrak{p}_{\lambda}^{i}\right)$ form an $A / \mathfrak{p}_{\lambda}^{i}$-module $\operatorname{ker}\left(\left.\varphi\left(\mathfrak{p}_{\lambda}^{i}\right)\right|_{k_{x}^{\text {sep }}}\right)$ which is free of rank $\leq n$. Thus, the rational Tate module

$$
V_{\lambda}\left(\varphi_{x}\right):=\operatorname{Hom}_{A_{\lambda}}\left(F_{\lambda}, \bigcup_{i \geq 0} \operatorname{ker}\left(\left.\varphi\left(\mathfrak{p}_{\lambda}^{i}\right)\right|_{k_{x}^{\mathrm{sep}}}\right)\right)
$$

is an $F_{\lambda}$-vector space of dimension $\leq n$. The dimension is equal to $n$ if and only if $\lambda \neq \lambda_{x}$. If $\varphi_{x}$ has special characteristic, the dimension of $V_{\lambda_{x}}\left(\varphi_{x}\right)$ is denoted by $n_{x}$. The deficiency $n-n_{x}$ is then $\geq 1$ and called the height of $\varphi_{x}$. If height $\left(\varphi_{x}\right)=1$, then $\varphi_{x}$ is called ordinary and $\varphi$ is said to have ordinary reduction at $x$.

Primarily we are interested in the Tate modules of $\varphi$, but they are related to those of the reductions $\varphi_{x}$, as follows. For any $i \geq 0$ the combined kernel $\operatorname{ker}\left(\left.\varphi\left(\mathfrak{p}_{\lambda}^{i}\right)\right|_{\boldsymbol{G}_{a, \mathbf{R}}}\right)$ is a finite flat commutative group scheme over $X$. Thus the elements of $K^{\text {sep }}$ annihilated by $\varphi\left(p_{\lambda}^{i}\right)$ already lie in the integral closure $R^{\text {sep }} \subset K^{\text {sep }}$ of $R$. Any lift of the point $x$ to a homomorphism $R^{\text {sep }} \rightarrow k_{x}^{\text {sep }}$ thus induces a natural restriction map

$$
V_{\lambda}(\varphi) \rightarrow V_{\lambda}\left(\varphi_{x}\right)
$$

which is surjective. For dimension reasons it is an isomorphism whenever $\lambda \neq \lambda_{x}$.

By construction the Tate module $V_{\lambda}\left(\varphi_{x}\right)$ carries a natural continuous action 
of the Galois group $\operatorname{Gal}\left(k_{x}^{\mathrm{sep}} / k_{x}\right)$. Also, by definition the above restriction map is equivariant under the decomposition group of $x$ inside $\operatorname{Gal}\left(K^{\text {sep }} / K\right)$. Note that the intertia group always acts trivially on the right hand side. Thus in the case $\lambda \neq \lambda_{x}$ we deduce that the representation of $\operatorname{Gal}\left(K^{\mathrm{sep}} / K\right)$ on $V_{\lambda}(\varphi)$ is unramified at $x$ and the action of the decomposition group is determined already by the arithmetic of $\varphi_{x}$.

In the following we choose a basis of $V_{\lambda}(\varphi)$, so that the Galois action corresponds to a continuous homomorphism

$$
\rho_{\lambda}: \operatorname{Gal}\left(K^{\mathrm{sep}} / K\right) \rightarrow \mathrm{GL}_{n}\left(F_{\lambda}\right)
$$

Drinfeld modules over finite fields. Let us apply the preceding remarks to a closed point $x \in X$. For $\lambda \neq \lambda_{x}$ the action of the decomposition group is determined by the image of the Frobenius element $\operatorname{Frob}_{x} \in \operatorname{Gal}\left(K^{\text {sep }} / K\right)$. We shall use the following fundamental facts.

Theorem 1.2. (cf. [15] Thm. 3.2 .3 (b).) The characteristic polynomial of $\rho_{\lambda}\left(\mathrm{Frob}_{x}\right)$ has coefficients in $A$ and is independent of $\lambda$, as long as $\lambda \neq \lambda_{x}, \infty$.

Let $\alpha_{1}, \ldots, \alpha_{n}$ be the eigenvalues of $\rho_{\lambda}\left(\right.$ Frob $\left._{x}\right)$ in an algebraic closure $\bar{F}$ of $F$. Consider an arbitrary place $\lambda_{1}$ of $F$ and an extension $\bar{\lambda}_{1}$ to $\bar{F}$. We normalize the valuation ord $\bar{\lambda}_{1}$ in such a way that a uniformizer at $\lambda_{1}$ in $F$ has valuation 1. The following can be said about the valuations of the $\alpha_{i}$. Let $F_{\lambda_{1}}$ denote the residue field at $\lambda_{1}$.

Theorem 1.3. (cf. Drinfeld [11] Prop. 2.1 or [15] Thm. 3.2.3 c-d.)

(a) We have ord $\bar{\lambda}_{1}\left(\alpha_{i}\right)=0$ for all $1 \leq i \leq n$ and $\lambda_{1} \neq \lambda_{x}, \infty$.

(b) For all $1 \leq i \leq n$ we have

$$
\operatorname{ord}_{\infty}\left(\alpha_{i}\right)=-\frac{1}{n} \cdot \frac{\left[k_{x} / \boldsymbol{F}_{p}\right]}{\left[\boldsymbol{F}_{\infty} / \boldsymbol{F}_{p}\right]}
$$

(c) We have

$$
\operatorname{ord}_{\bar{\lambda}_{x}}\left(\alpha_{i}\right) \begin{cases}=0 & \text { for precisely } n_{x} \text { of the } \alpha_{i}, \text { and } \\ >0 & \text { for the remaining } n-n_{x} \text { of the } \alpha_{i}\end{cases}
$$

The global Galois representation. In the rest of this section we list three crucial known facts which give lower bounds on the image of the Galois 
representation $\rho_{\lambda}$.

Theorem 1.4. $V_{\lambda}(\varphi)$ is a semisimple $F_{\lambda}\left[\mathrm{Gal}\left(K^{\mathrm{sep}} / K\right)\right]$-module.

Proof. This was proved by Taguchi ([31] Th.0.1) in the case that $K$ is a finite extension of $F$. We deduce from this the general case, as follows. First note that the semisimplicity of the action of a subgroup $\Delta \subset \mathrm{GL}_{n}\left(F_{\lambda}\right)$ depends only on the subalgebra $F_{\lambda} \Delta \subset M_{n \times n}\left(F_{\lambda}\right)$. Let $\Gamma_{\lambda}:=\rho_{\lambda}\left(\operatorname{Gal}\left(K^{\text {sep }} / K\right)\right)$.

Lemma 1.5. There exists an open normal subgroup $\Gamma_{1} \subset \Gamma_{\lambda}$ such that for any subgroup $\Delta \subset \Gamma_{\lambda}$ with $\Delta \Gamma_{1}=\Gamma_{\lambda}$ we have $F_{\lambda} \Delta=F_{\lambda} \Gamma_{\lambda}$.

Proof. Select elements $\gamma_{i} \in \Gamma_{\lambda}$ which form a basis of $F_{\lambda} \Gamma_{\lambda}$. If each of these is allowed to move in a small neighborhood, they still remain linearly independent. Thus there exists an open normal subgroup $\Gamma_{1} \subset \Gamma_{\lambda}$ such that the elements $\gamma_{i} \gamma_{1, i}$ form a basis of $F_{\lambda} \Gamma_{\lambda}$ for any choice of $\gamma_{1, i} \in \Gamma_{1}$. If $\Delta \Gamma_{1}=\Gamma_{\lambda}$, we can choose the $\gamma_{1, i}$ so that $\gamma_{i} \gamma_{1, i} \in \Delta$. The assertion follows.

Choose a subgroup $\Gamma_{1} \subset \Gamma_{\lambda}$ as in Lemma 1.5 and let $\tilde{K}$ be the corresponding finite Galois extension of $K$. Let $\tilde{X}$ be the normalization of $X$ in $\tilde{K}$, and denote the morphism $\tilde{X} \rightarrow X$ by $\pi$.

Lemma 1.6. There exists a point $x \in X$ so that

(a) $k_{x}$ is a finite extension of $F$, and

(b) $\pi^{-1}(x) \subset \tilde{X}$ is irreducible.

Proof. This is an easy consequence of the fact that $F$ is Hilbertian (cf. [13] Cor.12.8). As an alternative we give a direct proof using standard Bertini type arguments. Let $\boldsymbol{F}_{q} \subset K$ and $\boldsymbol{F}_{\tilde{q}} \subset \tilde{K}$ denote the respective fields of constants. Choose an infinite field $\boldsymbol{F}_{q} \subset k \subset \overline{\boldsymbol{F}}_{\tilde{q}}$ with $k \cap \boldsymbol{F}_{\tilde{q}}=\boldsymbol{F}_{q}$, and put $\tilde{k}:=k \cdot \boldsymbol{F}_{\tilde{q}} \cong k \otimes_{\boldsymbol{F}_{q}} \boldsymbol{F}_{\tilde{q}}$. After shrinking $X$ we may choose a dominant quasi-finite morphism $f: X \rightarrow \boldsymbol{A}_{\boldsymbol{F}_{q}}^{d}$ where $d:=\operatorname{dim}(X)$. Consider the commutative diagram

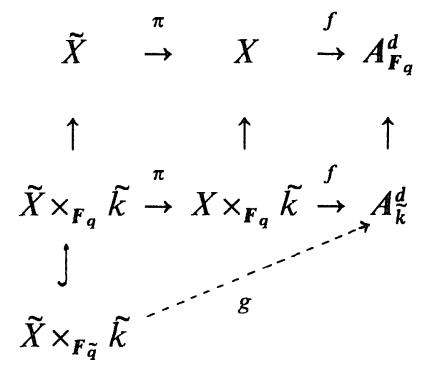


Note that $\tilde{X} \times_{F_{\tilde{q}}} \tilde{k}$ is geometrically irreducible over $\tilde{k}$, and the morphism $g$ is dominant and quasi-finite. Thus by repeatedly applying Jouanolou's version [21] Th. 6.3 of Bertini's theorem we find that $g^{-1}(\tilde{L})$ is geometrically irreducible of dimension 1 for every sufficiently generic affine line $\tilde{L} \subset A_{\tilde{k}}^{d}$.

Since $k$ is infinite, we may suppose that $\tilde{L}$ is already defined over $k$, i.e. that $\tilde{L}=L \times_{k} \tilde{k}$ for a line $L \subset A_{k}^{d}$. Then the diagram

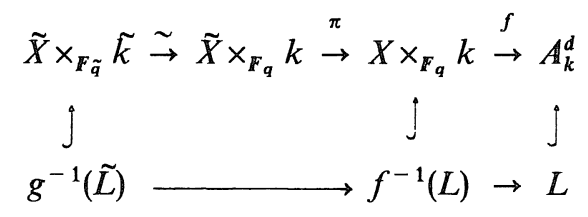

is cartesian. Let $x \in X$ be the image of the generic point of $f^{-1}(L)$. Then the irreducibility of $g^{-1}(\tilde{L})$ implies that of $\pi^{-1}(x)$, whence condition (b).

In order to satisfy condition (a) let us suppose that the first coordinate of $f$ factors through the morphism $X \rightarrow C \backslash\{\infty\}$. Consider the commutative diagram

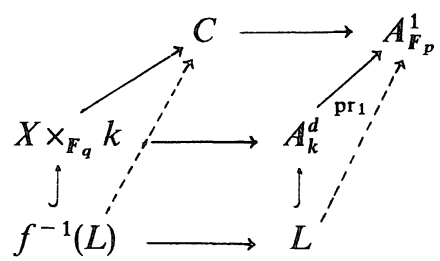

For generic $L$ the morphism $L \rightarrow A_{F_{p}}^{1}$ is non-constant. It follows that the morphism $f^{-1}(L) \rightarrow C$ is non-constant, which implies condition (a).

To prove Theorem 1.4 we choose $x$ as in Lemma 1.6 and let $\Delta_{\lambda}$ be the image of $\operatorname{Gal}\left(k_{x}^{\text {sep }} / k_{x}\right)$ in its representation on $V_{\lambda}\left(\varphi_{x}\right)$. Since $\varphi_{x}$ does not have characteristic $\lambda$, this Tate module can be identified with $V_{\lambda}(\varphi)$, which makes $\Delta_{\lambda}$ a subgroup of $\Gamma_{\lambda}$. Condition 1.6 (b) now means that $\Delta_{\lambda} \Gamma_{1}=\Gamma_{\lambda}$. Thus from Lemma 1.5 we deduce $F_{\lambda} \Delta_{\lambda}=F_{\lambda} \Gamma_{\lambda}$. On the other hand, by Lemma 1.6 
(a) and the theorem of Taguchi ([31] Th. 0.1) the ring on the left hand side acts semisimply. This proves Theorem 1.4 .

The next result characterizes the commutant of the image of Galois. The definition of Tate modules shows that the endomorphism ring of $\varphi$ acts on the Tate module by a natural homomorphism

$$
\operatorname{End}_{K}(\varphi) \otimes_{A} F_{\lambda} \rightarrow \operatorname{End}_{F_{\lambda}}\left(V_{\lambda}(\varphi)\right)
$$

This action commutes with the action of $\operatorname{Gal}\left(K^{\text {sep }} / K\right)$.

Theorem 1.7. (The "Tate conjecture": see Taguchi [32], resp. Tamagawa [33].) The natural map

$$
\operatorname{End}_{K}(\varphi) \otimes{ }_{A} F_{\lambda} \rightarrow \operatorname{End}_{F_{\lambda}\left[\operatorname{Gal}\left(K^{\operatorname{sep} / K}\right)\right]}\left(V_{\lambda}(\varphi)\right)
$$

is an isomorphism.

The last ingredient is the characterization of the determinant of the Galois representation.

Theorem 1.8. Let $A_{F}^{f}$ denote the adeles of $F$ outside the place $\infty$. Then the image of the composite homomorphism

$$
\mathrm{Gal}\left(K^{\mathrm{sep}} / K\right) \rightarrow \mathrm{GL}_{n}\left(A_{F}^{f}\right) \stackrel{\text { det }}{\rightarrow} \mathrm{GL}_{1}\left(A_{F}^{f}\right)
$$

is open.

Proof. If $n=1$, then $\varphi$ can be defined already over a finite extension of $F$. Thus in this case the openness follows, essentially, from the abelian class field theory of $F$ : see Hayes [19] Thm. 12.3 and Thm. 16.2 or [18] Thm. 9.2. (The result goes back to Drinfeld [10] §8 Thm.1, cf. also Goss [17] §7.7). For arbitrary $n$ one can construct a "determinant" Drinfeld module $\psi: A \rightarrow \operatorname{End}_{K}\left(G_{a}\right)$ of rank 1 whose Tate modules are isomorphic to the highest exterior powers of the Tate modules of $\varphi$. In other words, for every $\lambda \neq \infty$ one can define an isomorphism $V_{\lambda}(\psi) \cong \wedge_{F_{\lambda}}^{n} V_{\lambda}(\varphi)$ which is $\operatorname{Gal}\left(K^{\text {sep }} / K\right)$-equivariant (see Anderson [1], or Goss [16] Ex. 2.6.3). Thus the assertion reduces to the case $n=1$. 


\section{§2. Openness of the Image of Galois}

The aim of this section is to prove Theorems 0.1 and 0.2 of the introduction. We first assume that $\operatorname{End}_{\bar{K}}(\varphi)=A$; this assumption will remain in force until we turn to Theorem 0.2 at the end of the section. For any place $\lambda \neq \infty$ of $F$ we abbreviate

$$
\Gamma_{\lambda}:=\rho_{\lambda}\left(\operatorname{Gal}\left(K^{\mathrm{sep}} / K\right)\right) \subset \mathrm{GL}_{n}\left(F_{\lambda}\right)
$$

Let $G_{\lambda} \subset \mathrm{GL}_{n, F_{\lambda}}$ denote the Zariski closure of $\Gamma_{\lambda}$. Later on we shall see that $G_{\lambda}=\mathrm{GL}_{n, F_{\lambda}}$, but for the moment we do not even know whether $G_{\lambda}$ is connected. Let $G_{\lambda}^{\circ}$ denote its identity component. Then $G_{\lambda}^{\circ}\left(F_{\lambda}\right) \cap \Gamma_{\lambda}$ is the image of $\operatorname{Gal}\left(K^{\text {sep }} / K^{\prime}\right)$ for some finite extension $K^{\prime}$ of $K$ in $K^{\text {sep }}$. By Theorems 1.4 and 1.7 applied to $K^{\prime}$ in place of $K$ we know that $\mathrm{Gal}\left(K^{\mathrm{sep}} / K^{\prime}\right)$ acts absolutely irreducibly on the Tate module. It follows that the tautological representation of $G_{\lambda}^{\circ}$ is also absolutely irreducible. In particular, this implies that $G_{\lambda}^{\circ}$ is a reductive group (cf. Fact A.1 of the Appendix A).

For any closed point $x \in X$ we define

$$
a_{x}:=\operatorname{tr}\left(\rho_{\lambda}\left(\operatorname{Frob}_{x}\right)\right) \cdot \operatorname{tr}\left(\rho_{\lambda}\left(\operatorname{Frob}_{x}\right)^{-1}\right) .
$$

By Theorem 1.2 this is an element of $F$ which depends only on $x$, as long as $\lambda \neq \lambda_{x}, \infty$. As $x$ varies, these elements capture enough arithmetic information for all our purposes. First, by an adaptation of the argument in [24] Prop. 2.7 we obtain the following sufficient criterion for ordinary reduction.

Lemma 2.1. Assume

(a) $\left[k_{x} / F_{\lambda_{x}}\right]=1$, and

(b) $a_{x}$ is not a constant function in $F$, if $n \geq 2$.

Then $\varphi$ has ordinary reduction at $x$.

Proof. In the case $n=1$ there is nothing to prove, so we assume $n \geq 2$. If $\alpha_{1}, \cdots, \alpha_{n} \in \bar{F}$ are the eigenvalues of $\rho_{\lambda}\left(\right.$ Frob $\left._{x}\right)$, we have

$$
a_{x}=\left(\sum_{i=1}^{n} \alpha_{i}\right) \cdot\left(\sum_{i=1}^{n} \alpha_{i}^{-1}\right)=\sum_{i, j=1}^{n} \frac{\alpha_{i}}{\alpha_{j}}
$$

Now Theorem 1.3 has the following consequences. First the term $\alpha_{i} / \alpha_{j}$ is a unit at all places of $\bar{F}$ not dividing $\lambda_{x}$. Therefore $a_{x}$ is integral at these places. Next 
we have

$$
\operatorname{ord}_{\bar{\lambda}_{1}}\left(\prod_{i=1}^{n} \alpha_{i}\right)= \begin{cases}0 & \text { for } \lambda_{1} \neq \lambda_{x}, \infty \\ -\left[k_{x} / \boldsymbol{F}_{p}\right] /\left[\boldsymbol{F}_{\infty} / \boldsymbol{F}_{p}\right] & \text { for } \lambda_{1}=\infty\end{cases}
$$

where $\lambda_{1}$ is an arbitrary place of $F$. Since $\Pi_{i=1}^{n} \alpha_{i}$ is an element of $F$, the product formula implies

$$
\operatorname{ord}_{\bar{\lambda}_{x}}\left(\prod_{i=1}^{n} \alpha_{i}\right)=\left[k_{x} / \boldsymbol{F}_{\lambda_{x}}\right]
$$

By assumption (a) this value is equal to 1 . Suppose now that height $\left(\varphi_{x}\right)=n-n_{x}>1$. Then Theorem 1.3 (c) implies that $0 \leq \operatorname{ord}_{\bar{\lambda}_{x}}\left(\alpha_{i}\right)<1$ for all $i$. It follows that $\operatorname{ord}_{\bar{\lambda}_{x}}\left(\alpha_{i} / \alpha_{j}\right)>-1$ and hence $\operatorname{ord}_{\bar{\lambda}_{x}}\left(a_{x}\right)>-1$. Since $a_{x}$ is an element of $F$, its valuation is an integer, so $a_{x}$ is integral at $\lambda_{x}$. Thus we have shown that $a_{x}$ is integral at all places of $F$, contradicting assumption (b).

Lemma 2.2. The set of closed points $x \in X$ satisfying condition (b) of Lemma 2.1 has Dirichlet density $>0$.

Proof. Fix a place $\lambda \neq \infty$ of $F$, and recall that the given representation of $G_{\lambda}^{\circ}$ is absolutely irreducible. We may assume that $n \geq 2$. Then Proposition A.2 of the Appendix A implies that the morphism

$$
G_{\lambda}^{\circ} \rightarrow A_{F_{\lambda}}^{1}, \quad g \mapsto \operatorname{tr}(g) \cdot \operatorname{tr}\left(g^{-1}\right)
$$

is non-constant. Since the constant field of $F$ is finite, we deduce that the elements $g \in G_{\lambda}$ for which $\operatorname{tr}(g) \cdot \operatorname{tr}\left(g^{-1}\right)$ lies in this constant field form a Zariski closed proper subset. Let us call it $Z_{\lambda}$. By the definition of $G_{\lambda}$ as Zariski closure of $\Gamma_{\lambda}$, the intersection $Z_{\lambda}\left(F_{\lambda}\right) \cap \Gamma_{\lambda}$ is a proper closed subset of $\Gamma_{\lambda}$. With the Čebotarev density theorem (see Theorem B.9 of the Appendix B) we conclude that the set of closed points $x \in X$ with $\rho_{\lambda}\left(\right.$ Frob $\left._{x}\right) \notin Z_{\lambda}$ has Dirichlet density $>0$, as desired.

By Proposition B.8 of the Appendix B, applied to $Y=C$, the set of closed points $x \in X$ satisfying condition (a) of Lemma 2.1 has Dirichlet density 1. Combining this with Lemmas $2.1-2$ we obtain a first approximation to Theorem 0.3 . 
Corollary 2.3. The set of closed points $x \in X$ where $\varphi$ has ordinary reduction has Dirichlet density $>0$.

The set of $a_{x}$ also enjoys the following property, which will be needed below.

Proposition 2.4. Consider any Zariski open dense sebset $U \subset X$. If $n \geq 2$, then $F$ is the field generated by the elements $a_{x}$ for all closed points $x \in U$.

Proof. Let $E \subset F$ be the subfield generated by the $a_{x}$ in question. By Lemma 2.2, combined with Proposition B.7 (f) of the Appendix $\mathbb{B}$, some such $a_{x}$ is non-constant. Thus $E$ has transcendence degree 1 over $\mathbb{F}_{p}$, and therefore $[F / E]$ is finite. For any closed point $x \in U$ let $\mu_{x}$ denote the place of $E$ below $\lambda_{x}$. Let $D$ be the smooth projective curve with function field $E$, and apply Proposition B.8 of the Appendix B to the composite morphism $U \rightarrow C \rightarrow D$. We find that the set of $x \in U$ for which $\left[k_{x} / \mathbb{F}_{\mu_{x}}\right]=1$ has Dirichlet density 1 . Using Lemma 2.2 again we may choose $x \in U$ such that $a_{x}$ is non-constant and $\left[k_{x} / \mathbb{F}_{\mu_{x}}\right]=1$.

As in the proof of Lemma 2.1 we see that, as an element of $F$, the function $a_{x}$ has a unique pole at $\lambda_{x}$ and this pole is simple. But $a_{x}$ is contained in $E$, so there it must have a simple pole at $\mu_{x}$, and $\lambda_{x}$ is unramified over $\mu_{x}$. The choice of $x$ implies that $\left[F_{\lambda_{x}} / F_{\mu_{x}}\right]=1$, so the local degree $\left[F_{\lambda_{x}} / E_{\mu_{x}}\right]$ is equal to 1 . On the other hand, going back to $F$ we find that $a_{x}$ has a pole at every place dividing $\mu_{x}$. Thus $\lambda_{x}$ is the only place of $F$ above $\mu_{x}$. It follows that the global degree $[F / E]$ is equal to the local degree, i.e. $=1$, as desired.

Next we relate the valuations of the Frobenius eigenvalues to information about the algebraic groups $G_{\lambda}$. Consider a closed point $x \in X$ and choose an element $t_{x} \in \mathrm{GL}_{n}(F)$ whose characteristic polynomial coincides with that of $\rho_{\lambda}\left(\right.$ Frob $\left._{x}\right)$. As the characteristic of $F$ is non-zero, some positive power of $t_{x}$ is semisimple and lies in a unique conjugacy class. Let $T_{x} \subset \mathrm{GL}_{n, F}$ be the Zariski closure of the subgroup generated by $t_{x}$. By construction the identity component of $T_{x}$ is a torus, called Frobenius torus (following Serre, cf. [28], [4]).

Lemma 2.5. If $\varphi$ has ordinary reduction at $x$, then $T_{x}$ possesses a cocharacter over $\bar{F}$ which in the given representation has weight 1 with multiplicity 1 , and weight 0 with multiplicity $n-1$. 
Proof. The character group $X^{*}\left(T_{x}\right):=\operatorname{Hom}\left(T_{x} \times_{F} \bar{F}, G_{m}\right)$ is related to the cocharacter group by a canonical isomorphism $Y_{*}\left(T_{x}\right):=\operatorname{Hom}\left(G_{m}, T_{x} \times_{F} \bar{F}\right)$ $\cong \operatorname{Hom}\left(X^{*}\left(T_{x}\right), Z\right)$. Thus, with $\bar{\lambda}_{x}$ as in Theorem 1.3, the linear form

$$
X^{*}\left(T_{x}\right) \rightarrow \mathbb{Q}, \quad \chi \mapsto \operatorname{ord}_{\bar{\lambda}_{x}}\left(\chi\left(t_{x}\right)\right)
$$

defines an element of $Y_{*}\left(T_{x}\right) \otimes Q$. By Theorem 1.3 (c) its weights in the given representaion are 0 with multiplicity $n_{x}=n-1$, and some positive value with multiplicity 1. After rescaling this element so that the positive weight is 1 , all its weights are integral, so we obtain an element of $Y_{*}\left(T_{x}\right)$ with the desired properties.

Now we have collected enough information about $G_{\lambda}$ to be able to prove

Proposition 2.6. For any $\lambda \neq \infty$ we have $G_{\lambda}=\mathrm{GL}_{n, F_{\lambda}}$.

Proof. It is enough to prove $G_{\lambda}^{\circ}=\mathrm{GL}_{n, F_{\lambda}}$. Recall that the tautological representation of this group is absolutely irreducible. By Corollary 2.3 we may choose a closed point $x \in X$ with $\lambda_{x} \neq \lambda$, such that $\varphi$ has ordinary reduction at $x$. Then the semisimple parts of $t_{x}$ and $\rho_{\lambda}\left(\right.$ Frob $\left._{x}\right)$ are conjugate in $\mathrm{GL}_{n}\left(F_{\lambda}\right)$. Therefore, the group $T_{x} \times_{F} \quad F_{\lambda}$ is conjugate to an algebraic subgroup of $G_{\lambda}$. It follows that $G_{\lambda}$ possesses a cocharacter over $\bar{F}_{\lambda}$ with the same weights as in Lemma 2.5. Since any cocharacter factors through the identity component, the same follows for the group $G_{\lambda}^{\circ}$. The assertion now follows from Proposition A.3 of the Appendix A.

After these preparations we are ready to prove Theorem 0.1. Let us abbreviate $F_{\Lambda}:=\oplus_{\lambda \in \Lambda} F_{\lambda}$. Let $\Gamma_{\Lambda}$ denote the image of $\operatorname{Gal}\left(K^{\text {sep }} / K\right)$ in $\mathrm{GL}_{n}\left(F_{\Lambda}\right)=\Pi_{\lambda \in \Lambda} \mathrm{GL}_{n}\left(F_{\lambda}\right)$, and $\Gamma_{\Lambda}^{\prime}$ the closure of its commutator subgroup. By Theorem 1.8 it suffices to show that $\Gamma_{\Lambda}^{\prime}$ is open in $\operatorname{SL}_{n}\left(F_{\Lambda}\right)$. We may assume that $n \geq 2$ since otherwise there is nothing to prove.

By Proposition 2.6 we know already that the image of $\Gamma_{\Lambda}$ in $\operatorname{PGL}_{n}\left(F_{\lambda}\right)$ is Zariski dense for each $\lambda \in \Lambda$. We also need to know that the coefficients of $\Gamma_{\Lambda}$ in the adjoint representation of $\mathrm{PGL}_{n, F_{\Lambda}}$ cannot be made to lie in a proper subring of $F_{\Lambda}$. This is achieved by the following lemma. Let $\mathcal{O} \subset F_{\Lambda}$ be the closure of the subring generated by 1 and by $\operatorname{tr}\left(\operatorname{Ad}_{\mathrm{PGL}_{n}}\left(\Gamma_{\Lambda}\right)\right)$.

Lemma 2.7. $F_{\Lambda}$ is the total ring of quotients of $\mathcal{O}$. 
Proof. Let $U \subset X$ be the Zariski open subset consisting of all points $x$ with $\lambda_{x} \notin \Lambda$. Then for any $x \in U$ and any $\lambda \in \Lambda$ we have $\operatorname{tr}\left(\operatorname{Ad}_{\mathrm{PGL}_{n}}\left(\rho_{\lambda}\left(\operatorname{Frob}_{x}\right)\right)\right)$ $=a_{x}-1$. It follows that $a_{x}$, diagonally embedded in $F_{\Lambda}$, lies in $\mathcal{O}$. Now Proposition 2.4 implies that $F$, also diagonally embedded, is contained in the total ring of quotients of $\mathcal{O}$. As $F$ is dense in $F_{\Lambda}$, the assertion follows.

The rest of the argument is pure group theory, though quite involved. The general problem is to show that compact subgroups of semisimple groups over local fields are in some sense essentially algebraic. This was achieved by the author in a separate article. The following special case is enough for our present purposes.

Theorem 2.8. (Combine [25] Main Theorem 0.2 with Prop. 0.4 (c).) For any $i$ in a finite index set I let $F_{i}$ be a local field, and put $F_{I}=\oplus_{i \in I} F_{i}$. Let $n \geq 2$ and consider a compact subgroup $\Gamma \subset \mathrm{GL}_{n}\left(F_{I}\right)=\Pi_{i \in I} \mathrm{GL}_{n}\left(F_{i}\right)$ whose image in each $\operatorname{PGL}_{n}\left(F_{i}\right)$ is Zariski dense. Let $\mathcal{O} \subset F_{I}$ be the closure of the subring generated by 1 and by $\operatorname{tr}\left(\operatorname{Ad}_{\mathrm{PGL}_{n}}(\Gamma)\right)$, and assume that $F_{I}$ is the total ring of quotients of 0 . Then the closure of the commutator subgroup of $\Gamma$ is open in $\mathrm{SL}_{n}\left(F_{I}\right)=\Pi_{i \in I} \mathrm{SL}_{n}\left(F_{i}\right)$.

With Lemma 2.7 and Theorem 2.8, the proof of Theorem 0.1 is complete.

Now we turn to Theorem 0.2 , which is, in fact, easily deduced from Theorem 0.1. As in $\S 1$ we put $A^{\prime}:=\operatorname{End}_{\bar{K}}(\varphi)$ and $F^{\prime}:=\operatorname{Quot}\left(A^{\prime}\right)$, and let $\tilde{A}^{\prime}$ be the normalization of $A^{\prime}$ in $F^{\prime}$. Since every isogeny induces an isomorphism on Tate modules, we may replace $\varphi$ by the isogenous Drinfeld module of Proposition 1.1. Thus we may assume that $A^{\prime}=\tilde{A}^{\prime}$. By the assumption in Theorem 0.2 we have $\operatorname{End}_{K}(\varphi)=A^{\prime}$. Let $\varphi^{\prime}: A^{\prime} \rightarrow \operatorname{End}_{K}\left(G_{a}\right)$ be the tautological extension of $\varphi$. This is a Drinfeld module of rank $n^{\prime}$, where $n=\operatorname{rank}_{A}\left(A^{\prime}\right) \cdot n^{\prime}$. For any place $\lambda \neq \infty$ of $F$ the definition of Tate modules gives a natural isomorphism

$$
V_{\lambda}(\varphi) \cong \underset{\lambda^{\prime} \mid \lambda}{\bigoplus} V_{\lambda^{\prime}}\left(\varphi^{\prime}\right)
$$

which commutes with the actions of both $A^{\prime}$ and $\operatorname{Gal}\left(K^{\text {sep }} / K\right)$. Now let $\Lambda^{\prime}$ be the set of places of $F^{\prime}$ lying over some $\lambda \in \Lambda$. Then the above isomorphism 
and the Galois representations associated to $\varphi$ and $\varphi^{\prime}$ induce a commutative diagram

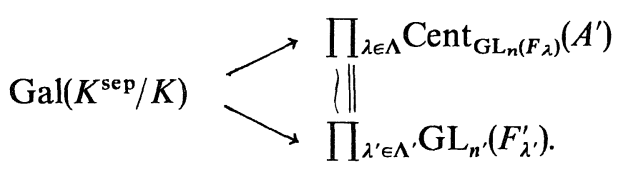

Thus Theorem 0.2 is reduced to Theorem 0.1 for the lower homomorphism.

\section{§3. Occurrences of a Given Newton Polygon}

The aim of this section is to determine the Dirichlet density of the set of closed points in $X$ where the reduction of $\varphi$ has a given Newton polygon, i.e. has prescribed height. The result has been summarized in Theorem 0.3. As before we put $A^{\prime}:=\operatorname{End}_{\bar{K}}(\varphi)$ and $F^{\prime}:=$ Quot $\left(A^{\prime}\right)$, and let $\tilde{A}^{\prime}$ be the normalization of $A^{\prime}$ in $F^{\prime}$. After replacing $\varphi$ by the isogenous Drinfeld module of Proposition 1.1 we may assume $A^{\prime}=\tilde{A}^{\prime}$.

Let $K^{\prime} \subset \bar{K}$ be the finite extension of $K$ generated by the coefficients of all endomorphisms in $\operatorname{End}_{\bar{K}}(\varphi)$. The Tate conjecture (Theorem 1.7) implies that all endomorphisms over $\bar{K}$ are defined already over $K^{\text {sep }}$. Thus $K^{\prime}$ is separable and Galois over $K$. By construction the Galois group $\Delta:=\operatorname{Gal}\left(K^{\prime} / K\right)$ acts also on $F^{\prime}$, and by the Tate conjecture this action is faithful. Let $\varphi^{\prime}: A^{\prime} \rightarrow \operatorname{End}_{K^{\prime}}\left(G_{a}\right)$ be the tautological extension of $\varphi$. This is a Drinfeld module of rank $n^{\prime}$, where $n=\left[F^{\prime} / F\right] \cdot n^{\prime}$. We have the following commutative diagram:

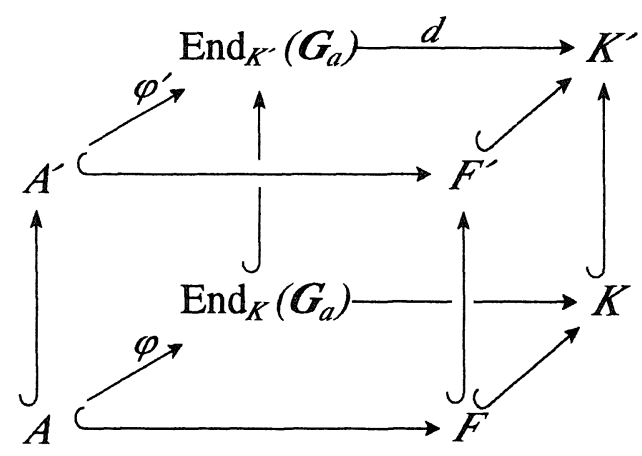


Here the homomorphism $d: \operatorname{End}_{K^{\prime}}\left(G_{a}\right) \rightarrow K^{\prime}$ denotes the derivative at 0 , i.e. the action on the Lie algebra of $\mathbb{G}_{a}$. The whole diagram is compatible with the obvious actions of $\Delta$. In particular the inclusion $F^{\prime} \hookrightarrow K^{\prime}$ is $\Delta$-equivariant, so we have $K^{\prime}=F^{\prime} K$. One should be aware that $F^{\prime} / F$ might be inseparable although, as we have seen, the extension $K^{\prime} / K$ is always separable.

Now let $X$ be any model of $K$ of finite type over Spec $\mathbb{F}_{q}$. We may suppose that $\varphi$ has good reduction everywhere on $X$. Let $X^{\prime}$ be the normalization of $X$ in $K^{\prime}$. Then $\varphi^{\prime}$ has good reduction everywhere on $X^{\prime}$. Consider a closed point $x^{\prime} \in X^{\prime}$ with image $x \in X$, and let $\lambda_{x^{\prime}}^{\prime}$ denote the place of $F^{\prime}$ below $x^{\prime}$. The height of the reductions $\varphi_{x}$ and $\varphi_{x^{\prime}}^{\prime}$ are related as follows.

Lemma 3.2. We have

$$
\operatorname{height}\left(\varphi_{x}\right)=\left[F_{\lambda^{\prime} x^{\prime}}^{\prime} / F_{\lambda_{x}}\right] \cdot \operatorname{height}\left(\varphi_{x^{\prime}}^{\prime}\right)
$$

Proof. By the definition of Tate modules we have an isomorphism

$$
V_{\lambda_{x}}\left(\varphi_{x}\right) \cong \underset{\lambda^{\prime} \mid \lambda_{x}}{\bigoplus} V_{\lambda^{\prime}}\left(\varphi_{x^{\prime}}^{\prime}\right)
$$

Thus we can calculate

$$
\begin{aligned}
\operatorname{height}\left(\varphi_{x}\right) & =n-\operatorname{dim}_{F_{\lambda_{x}}}\left(V_{\lambda_{x}}\left(\varphi_{x}\right)\right) \\
& =\sum_{\lambda^{\prime} \mid \lambda_{x}}\left[F_{\lambda^{\prime}}^{\prime} / F_{\lambda_{x}}\right] \cdot\left(n^{\prime}-\operatorname{dim}_{F_{\lambda^{\prime}}}\left(V_{\lambda^{\prime}}\left(\varphi_{x^{\prime}}^{\prime}\right)\right)\right) \\
& =\left[F_{\lambda_{x^{\prime}}}^{\prime} / F_{\lambda_{x}}\right] \cdot\left(n^{\prime}-\operatorname{dim}_{F_{\lambda^{\prime}}^{\prime}}\left(V_{\lambda^{\prime} x^{\prime}}\left(\varphi_{x^{\prime}}^{\prime}\right)\right)\right) \\
& =\left[F_{\lambda_{x^{\prime}}^{\prime}}^{\prime} / F_{\lambda_{x}}\right] \cdot \operatorname{height}\left(\varphi_{x^{\prime}}^{\prime}\right),
\end{aligned}
$$

as desired.

Let $p^{e}$ denote the degree of the totally inseparable part of the extension $F^{\prime} / F$. Then the first factor in Lemma 3.2 is always divisible by $p^{e}$. This already proves part (a) of Theorem 0.3 . For part (b) we may replace $X$ by an arbitrary Zariski dense open subset, since by Proposition B.7 (f) of the Appendix B this does not change Dirichlet densities. For instance, we may suppose that $X$ is normal. As $\varphi^{\prime}$ has good reduction at any point of $X^{\prime}$, the inclusion $A^{\prime} \hookrightarrow K^{\prime}$ corresponds to a morphism $X^{\prime} \rightarrow \operatorname{Spec} A^{\prime}$. Let $A^{\prime \Delta}$ denote the subring of $\Delta$-invariants in $A^{\prime}$, then we have in fact a commutative diagram 


$$
\begin{array}{ccc}
X^{\prime} \rightarrow & \operatorname{Spec} A^{\prime} \\
\downarrow & \downarrow \\
X & \rightarrow & \operatorname{Spec} A^{\prime \Delta} \\
& & \downarrow \\
& & \operatorname{Spec} A .
\end{array}
$$

By construction the upper two vertical morphisms are Galois coverings with Galois group $\Delta$. Thus after shrinking $X$ and $X^{\prime}$ we may assume that $X^{\prime}$ is étale over $X$ and that the upper rectangle in Diagram (3.3) is cartesian. Now we can analyze more closely the first factor in Lemma 3.2.

Lemma 3.4. We have

$$
p^{e} \cdot\left[k_{x^{\prime}} / k_{x}\right] \mid\left[F_{\lambda^{\prime} x^{\prime}}^{\prime} / F_{\lambda_{x}}\right],
$$

with equality for $x$ in a set of points of Dirichlet density 1.

Proof. As Diagram (3.3) is cartesian, we have $k_{x^{\prime}}=\boldsymbol{F}_{\lambda^{\prime} x} k_{x}$. This shows that $\left[k_{x^{\prime}} / k_{x}\right]$ divides $\left[\boldsymbol{F}_{\lambda^{\prime} x^{\prime}} / \boldsymbol{F}_{\lambda_{x}}\right]$, with equality if $\left[k_{x} / \boldsymbol{F}_{\lambda_{x}}\right]=1$. Note that by Proposition B.8 of the Appendix B, applied to $Y=C$, this last condition holds on a set of points $x$ of Dirichlet density 1. On the other hand the ramification degree of $F_{\lambda^{\prime} x^{\prime}}^{\prime}$ over $F_{\lambda_{x}}$ is always divisible by $p^{e}$, with equality outside a Zariski closed proper subset of $X$. The assertion follows.

To bound the second factor in Lemma 3.2 we follow the same procedure as in $\S 2$. For the present purposes it is enough to work with the element

$$
b_{x^{\prime}}:=\operatorname{tr}\left(\rho_{\lambda^{\prime}}\left(\mathrm{Frob}_{x^{\prime}}\right)^{-1}\right) \in F^{\prime},
$$

where $\rho_{\lambda^{\prime}}$ denotes the Galois representation associated to the Drinfeld module $\varphi^{\prime}$ for any sufficiently general place $\lambda^{\prime}$ of $F^{\prime}$.

\section{Lemma 3.5. Assume}

(a) $\left[k_{x^{\prime}} / F_{\lambda^{\prime} x^{\prime}}^{\prime}\right]=1$, and

(b) $b_{x^{\prime}}$ is not a constant function in $F^{\prime}$.

Then height $\left(\varphi_{x}^{\prime}\right)=1$. 
Proof. The proof is essentially that of Lemma 2.1, with $\varphi$ replaced by $\varphi^{\prime}$. By the same arguments as in 2.1 we find that $b_{x^{\prime}}$ is integral at all places of $F^{\prime}$ other than $\lambda_{x^{\prime}}^{\prime}$, and its valuation at $\lambda_{x^{\prime}}^{\prime}$ is $>-1$ if $\varphi_{x^{\prime}}^{\prime}$ is not ordinary. In that case $b_{x^{\prime}}$ must be constant, contradicting assumption (b).

Lemma 3.6. The set of closed points $x \in X$ for which condition (a) of Lemma 3.5 holds has Dirichlet density 1.

Proof. Since Diagram (3.3) is cartesian, we have $\left[k_{x^{\prime}} / \mathbb{F}_{\lambda^{\prime} x^{\prime}}\right] \mid\left[k_{x} / F_{\lambda_{x}}\right]$ for all $x$. By Proposition B.8 the latter index is 1 for a set of $x$ of density 1 .

Lemma 3.7. The set of closed points $x \in X$ for which condition (b) of Lemma 3.5 holds has Dirichlet density 1.

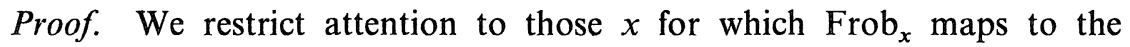
conjugacy class of a fixed element $\delta \in \Delta$. The choice of $x^{\prime}$ determines the image of Frob $_{x}$ in its conjugacy class, so we may assume that Frob $b_{x}$ maps to $\delta$ itself. Let $l$ denote the order of $\delta$.

Next fix a place $\lambda \neq \infty$ of $F$ which is maximally split in $F^{\prime}$. In other words, if $\tilde{F}$ denotes the maximal totally inseparable extension of $F$ inside $F^{\prime}$, then the unique place $\tilde{\lambda}$ of $\tilde{F}$ above $\lambda$ splits completely in $F^{\prime}$. Recall that $V_{\lambda}(\varphi)=\oplus_{\lambda^{\prime} \mid \lambda} V_{\lambda^{\prime}}\left(\varphi^{\prime}\right)$. Removing from $X$ the fiber above $\lambda$, the conditions on $x$ imply that Frob $x$ maps each $V_{\lambda^{\prime}}\left(\varphi^{\prime}\right)$ to $V_{\lambda^{\prime}}\left(\varphi^{\prime}\right)$.

Let us fix a place $\lambda^{\prime} \mid \lambda$. Then the Tate modules $V_{\lambda^{\prime} \delta^{\prime}}\left(\varphi^{\prime}\right)$ for $i \bmod l$ are $\widetilde{F}_{\tilde{\lambda}}$-vector spaces of dimension $n^{\prime}$ which are cyclically permuted by Frob $_{x}$. Let us choose bases for these Tate modules, not depending on $x$ (but, of course, on $\delta$ and $\lambda^{\prime}$ ). Then for each $i \bmod l$ the map

$$
V_{\lambda^{\prime} \delta^{i}}\left(\varphi^{\prime}\right) \rightarrow V_{\lambda^{\prime} \delta^{i+1}}\left(\varphi^{\prime}\right)
$$

induced by Frob $_{x}$ is given by a matrix $g_{i} \in \mathrm{GL}_{n^{\prime}}\left(\tilde{F}_{\tilde{\lambda}}\right)$. The assumptions on $x$ imply that Frob $_{x^{\prime}}=$ Frob $_{x}^{l}$, hence Frob $_{x^{\prime}}$ acts on $V_{\lambda^{\prime}}\left(\varphi^{\prime}\right)$ through the product $g_{l-1} \cdots g_{1} g_{0}$. It follows that

$$
b_{x^{\prime}}=\operatorname{tr}\left(\left(g_{l-1} \cdots g_{1} g_{0}\right)^{-1}\right) .
$$

On the other hand Theorem 0.1 applied to $\varphi^{\prime}$ and the set of places $\Lambda^{\prime}:=\left\{\lambda^{\prime \delta^{\prime}} \mid i \bmod l\right\}$ asserts that $\operatorname{Gal}\left(K^{\prime s e p} / K^{\prime}\right)$ acts on the above Tate modules 
through an open subgroup

$$
\Gamma_{\Lambda^{\prime}} \subset \prod_{i \bmod l} \mathrm{GL}_{n^{\prime}}\left(\tilde{F}_{\tilde{\lambda}}\right)
$$

Under the current assumptions on $x$ the tuple $\left(g_{0}, g_{1}, \cdots, g_{l-1}\right)$ associated to Frob ${ }_{x}$ runs through a certain coset $H_{\Lambda^{\prime}}$ under $\Gamma_{\Lambda^{\prime}}$.

Let $\tilde{m}$ denote the maximal ideal of the valuation ring in $\tilde{F}_{\tilde{\lambda}}$, and for every $j \geq 0$ let $K_{j}$ be the finite separable extension of $K^{\prime}$ corresponding to the subgroup

$$
\left\{\gamma \in \Gamma_{\Lambda^{\prime}} \mid \gamma \equiv \operatorname{id} \bmod \tilde{m}^{j}\right\}
$$

Let $X_{j}$ be the normalization of $X$ in $K_{j}$. Since the fiber above $\lambda$ was removed from $X$, the theory of moduli of Drinfeld modules with level structure implies that $X_{j}$ is étale over $X$. We want to apply the Čebotarev density theorem to this covering. Let $F^{\prime}$ be the field of constants in $F^{\prime}$. Then the condition $b_{x^{\prime}} \in \boldsymbol{F}^{\prime}+\tilde{\mathrm{m}}^{j}$ depends only on the behavior of $x$ in $K_{j}$. It suffices to prove that the proportion of those $x \in X$ which satisfy this condition goes to 0 as $j \rightarrow \infty$. This follows from Čebotarev (see Theorem B.9 of the Appendix B) and the following sublemma.

Sublemma 3.8. The volume of the subset

$$
\left\{\left(g_{0}, g_{1}, \cdots, g_{l-1}\right) \in H_{\Lambda^{\prime}} \mid \operatorname{tr}\left(\left(g_{l-1} \cdots g_{1} g_{0}\right)^{-1}\right) \in \boldsymbol{F}^{\prime}+\tilde{m}^{j}\right\}
$$

with respect to any given Haar measure on $\Pi_{i \bmod l} \mathrm{GL}_{n^{\prime}}\left(\tilde{F}_{\tilde{\lambda}}\right)$ goes to zero as $j \rightarrow \infty$.

Proof. The isomorphism

$$
\begin{aligned}
& \prod_{i \bmod l} \mathrm{GL}_{n^{\prime}}\left(\tilde{F}_{\tilde{\lambda}}\right) \stackrel{\sim}{\rightarrow} \prod_{i \bmod l} \mathrm{GL}_{n^{\prime}}\left(\tilde{F}_{\tilde{\lambda}}\right) \\
& \left(g_{0}, g_{1}, \cdots, g_{l-1}\right) \mapsto\left(\left(g_{l-1} \cdots g_{1} g_{0}\right)^{-1}, g_{1}, \cdots, g_{l-1}\right)
\end{aligned}
$$

maps Haar measure to Haar measure and $H_{\Lambda^{\prime}}$ to a compact subset $H^{\prime}$ which is invariant under some other open subgroup $\Gamma^{\prime} \subset \Pi_{i \bmod l} \mathrm{GL}_{n^{\prime}}\left(\tilde{F}_{\tilde{\lambda}}\right)$. It suffices to prove that the volume of the subset

$$
\left\{\left(h_{0}, g_{1}, \cdots, g_{l-1}\right) \in H^{\prime} \mid \operatorname{tr}\left(h_{0}\right) \in \boldsymbol{F}^{\prime}+\tilde{\mathrm{m}}^{j}\right\}
$$

goes to zero as $j \rightarrow \infty$. The condition on $h_{0}$ shows that this volume is a constant 
times $\operatorname{card}\left(\boldsymbol{F}^{\prime}\right)^{-j}$ for all $j \gg 0$. This proves Sublemma 3.8 and thus Lemma 3.7.

Proof of Theorem 0.3. Part (a) was proved already after Lemma 3.2. For part (b) note that Lemmas 3.2-7 imply height $\left(\varphi_{x}\right)=p^{e} \cdot\left[k_{x^{\prime}} / k_{x}\right]$ for all $x$ in a set of points of Dirichlet density 1. Moreover, $\left[k_{x^{\prime}} / k_{x}\right]$ is just the order of the image of Frob ${ }_{x}$ in $\Delta$. The Dirichlet density of the set of $x$ with fixed $\left[k_{x^{\prime}} / k_{x}\right]$ is given by the Čebotarev density theorem (Theorem B.9 of the Appendix B), yielding the desired formula of 0.3 (b).

\section{Appendix A. Ingredients from the Theory of Algebraic Groups}

In this appendix we consider a connected linear algebraic group $G \subset \mathrm{GL}_{n, L}$ where $L$ is a field and $n$ a positive integer, both arbitrary. We assume that $G$ acts absolutely irreducibly on the vector space $V:=L^{n}$. If $\bar{L}$ denotes an algebraic closure of $L$, this means that $G \times_{L} \bar{L}$ acts irreducibly on $V \otimes_{L} \bar{L}=\bar{L}^{n}$.

Fact $\mathbf{A} . \mathbb{1} . \quad G$ is reductive.

Proof. We must show that $G \times{ }_{L} \bar{L}$ is reductive. Without loss of generality we may assume that $L=\bar{L}$. Let $U$ denote the unipotent radical (i.e. the largest connected unipotent normal subgroup) of $G$. By the theorem of Lie-Kolchin (cf. Humphreys [20] §17.6) the subspace of $U$-invariants in $V$ is non-zero. By construction it is also $G$-stable, so by irreducibility it must be the whole space. Thus $U$ acts trivially on $V$, hence $U$ itself is trivial. This means that $G$ is reductive $([20] \S 19.5)$.

Proposition A.2. Let $A_{L}^{1}$ denote the affine line as algebraic variety over L. Suppose that $n \geq 2$. Then the morphism $f: G \rightarrow \mathbb{A}_{L}^{1}, g \mapsto \operatorname{tr}(g) \cdot \operatorname{tr}\left(g^{-1}\right)$ is non-constant.

Proof. After base extension we may assume that $L$ is algebraically closed. Choose a maximal torus and a Borel subgroup $T \subset B \subset G$. Since $V$ is an irreducible representation, it has a unique highest weight $\lambda$, which is dominant and occurs with multiplicity 1 ([20] $\S 31.3)$. Since $\operatorname{dim}_{L}(V)>1$, we must have $\lambda \neq 0$. Let $\lambda^{*}$ be the highest weight of the dual representation $V^{*}$. Then the weight $\lambda^{*}+\lambda$ occurs with multiplicity 1 in the representation $V^{*} \otimes_{L} V . \quad$ For any character $\chi$ of $T$ let $m_{\chi} \in \mathbb{Z}$ denote the multiplicity of $\chi$ as 
a weight on $V^{*} \otimes_{L} V . \quad$ Then we have $\left.f\right|_{T}=\Sigma_{\chi} m_{\chi} \cdot \chi . \quad$ By the linear independence of characters such a function is constant if and only if for every $\chi \neq 0$ the coefficient $m_{\chi}$ maps to 0 in $L$. Since $\lambda^{*}+\lambda \neq 0$ and $m_{\lambda *+\lambda}=1$, this function is non-constant. Therefore the original function $f$ is non-constant.

Proposition A.3. Suppose that there exists a cocharacter $\mu: G_{m, \bar{L}} \rightarrow G \times{ }_{L} \bar{L}$ which on $V \otimes_{L} \bar{L}$ has weight 1 with multiplicity 1 , and weight 0 with multiplicity $n-1$. Then $G=\mathrm{GL}_{n, L}$.

Proof. (A more general treatment of such situations is in Serre [27] §3, the assumption of characteristic zero being unnecessary. For convenience we give a full proof here.) Again we may assume that $L$ is algebraically closed. Since det $\circ \mu$ is non-trivial, it suffices to prove that the derived group $G^{\text {der }}$ is equal to $\mathrm{SL}_{n, L}$. This is obvious when $n=1$, so let us assume $n \geq 2$. First we show that $G^{\text {der }}$ is simple. If this is not the case, then $V$ is $\otimes$-decomposable under G. That is, there exists an isomorphism $V \cong\left(L^{n_{1}}\right) \otimes_{L}\left(L^{n_{2}}\right)$ with $n_{1}, n_{2}>1$ such that $G$ is contained in $\mathrm{GL}_{n_{1}, L} \cdot \mathrm{GL}_{n_{2}, L} \subset \mathrm{GL}_{n, L}$. We can write $\mu=\mu_{1} \otimes \mu_{2}$ for suitable cocharacters $\mu_{i}: G_{m, L} \rightarrow \mathrm{GL}_{n_{i}, L}$. If $\mu_{i}$ is scalar for some $i$, the multiplicity of each weight of $\mu$ on $V$ is divisible by $n_{i}$. If, on the other hand, both $\mu_{i}$ are non-scalar, one easily shows that $\mu$ possesses at least three distinct weights on $V$. In both cases we obtain a contradiction to our assumption on $\mu$. Thus $G^{\text {der }}$ is simple.

Next note that the weights of $\mu$ on $\mathrm{gI}_{n . L}$ are \pm 1 and 0 . Thus the weights on Lie $G^{\text {der }}$ are also among these values. Choose a maximal torus and a Borel subgroup such that $\mu\left(G_{m . L}\right) \subset T \subset B \subset G$, and let $W$ denote the Weyl group of $G$ with respect to $T$. By Bourbaki [2] chap. $8 \S 7.3$ the highest weight $\lambda$ of $V$ is minuscule. It follows that all the weights of $T$ in $V$ are conjugate to $\lambda$ under $W$. (The proof is the same as in characteristic zero: all weights are contained in the " $R$-saturation of $\lambda$ " of loc. cit.) Since $\lambda$ has multiplicity 1 , so do all the weights. Let us put $T$ in diagonal form. We may suppose that

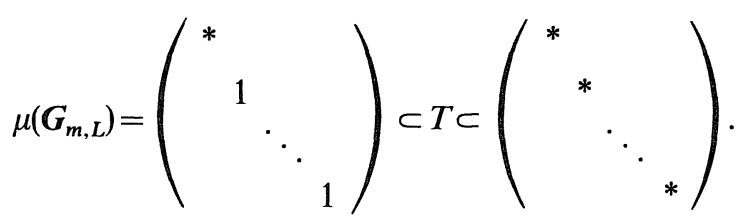

Since the weights are pairwise distinct and permuted transitively by $W$, the 
permutation representation $W \rightarrow S_{n}$ is transitive. Thus the $W$-conjugates of $\mu\left(G_{m . L}\right)$ altogether generate the torus $G_{m, L}^{n}$. It follows that $T=G_{m, L}^{n}$.

Now we know that $G^{\text {der }}$ is a simple semisimple subgroup of $\mathrm{SL}_{n . L}$ of equal rank. Therefore its root system is an irreducible closed root subsystem of $A_{n-1}$ of equal rank. It is easy to show that the only such subsystem is $A_{n-1}$ itself (cf. Dynkin [12] Table 9). We conclude that $G=\mathrm{GL}_{n, L}$, as desired.

\section{Appendix B. Dirichlet Density in Dimension $\geq 1$}

For lack of a suitable reference, this appendix describes a formalism of Dirichlet density for schemes of arbitrary dimension and establishes some of its main properties. If we are given a field $K$ which is finitely generated over its prime field, we must choose a model of finite type over $\operatorname{Spec} \mathbb{Z}$ in order to make sense of the statements below. However, Proposition B.7 (f) will show that the concept depends essentially only on $K$. The assertions will be stated regardless of the characteristic of $K$. But as the positive characteristic case is all that is needed in this article, any special arguments for the characteristic zero case will only be sketched. Everything here follows well-known arguments, as for instance those giving the equidistribution theorem of Deligne [7] $\mathrm{Th}$. 3.5.1 (see also Katz [22] Th. 3.6).

In the following all schemes will be of finite type over Spec $Z$. The set of closed points of such a scheme $X$ is denoted $|X|$. The residue field at $x \in|X|$ is denoted $k_{x}$ and its cardinality $q_{x}$. The following statement gives estimates for the number of closed points with a given residue field.

Proposition B.1. Let $f: X \rightarrow Y$ be a morphism of schemes of finite type over Spec $\mathbb{Z}$.

(a) Suppose that all fibers of $f$ have dimension $\leq \delta$. Then there exists a constant $C>0$ such that for all $y \in|Y|$ and all $n \geq 1$ we have

$$
\operatorname{card}\left\{x \in|X| \mid f(x)=y \text { and }\left[k_{x} / k_{y}\right]=n\right\} \leq \frac{1}{n} \cdot C \cdot q_{y}^{n \delta} .
$$

(b) Suppose that $f$ is surjective and all fibres are geometrically irreducible of dimension $\delta>0$. Then there exists a constant $C^{\prime}>0$ such that for all $y \in|Y|$ and all $n \geq 1$ we have

$$
\operatorname{card}\left\{x \in|X| \mid f(x)=y \text { and }\left[k_{x} / k_{y}\right]=n\right\} \geq \frac{1}{n} \cdot\left(q_{y}^{n \delta}-C^{\prime} \cdot q_{y}^{n\left(\delta-\frac{1}{2}\right)}\right) \text {. }
$$

Proof. Let $X_{y}$ denote the fiber of $f$ above $y$ and $k_{y}^{(n)}$ an extension 
of $k_{y}$ degree $n$. By Grothendieck's Lefschetz trace formula (see [6] Rapport Th. 3.2) we have

$$
\operatorname{card}\left(X_{y}\left(k_{y}^{(n)}\right)\right)=\sum_{i=0}^{2 \delta}(-1)^{i} \operatorname{tr}\left(\left.\operatorname{Frob}_{y}^{n}\right|_{H_{c}^{\prime}\left(X_{y} \times{ }_{k_{y}} \bar{k}_{y}, \mathbf{Q}_{1}\right)}\right)
$$

and by a theorem of Deligne (the "Weil conjecture", see [7] Th. 3.3.1) the eigenvalues of Frob $_{y}$ on $H_{c}^{i}\left(X_{y} \times{ }_{k_{y}} \bar{k}_{y}, Q_{l}\right)$ are algebraic numbers of complex absolute value $\leq q_{y}^{i / 2}$. Moreover, by constructibility and proper base change (see Deligne [6] Arcata) the total number of eigenvalues is bounded independently of $y$. This implies that

$$
\operatorname{card}\left(X_{y}\left(k_{y}^{(n)}\right)\right) \leq C \cdot q_{y}^{n \delta}
$$

for all $y$ and $n$, with a fixed constant $C>0$. Since any point $x \in|X|$ with $f(x)=y$ and $\left[k_{x} / k_{y}\right]=n$ corresponds to precisely $n$ primitive points in $X_{y}\left(k_{y}^{(n)}\right)$, this implies assertion (a). To prove (b) we first estimate the number of non-primitive points in $X_{y}\left(k_{y}^{(n)}\right)$.

Lemma B.3. In the situation of Proposition B.1 (b) let $X_{y}\left(k_{y}^{(n)}\right)^{\mathrm{imprim}} \subset X_{y}\left(k_{y}^{(n)}\right)$ denote the subset of those points which are defined over some proper subfield of $k_{y}^{(n)}$. Then for all $y \in|Y|$ and all $n \geq 1$ we have

$$
\operatorname{card}\left(X_{y}\left(k_{y}^{(n)}\right)^{\mathrm{imprim}}\right) \leq 2 C \cdot q_{y}^{n \delta / 2} .
$$

Proof. Using (B.2) we calculate

$$
\begin{aligned}
\operatorname{card}\left(X_{y}\left(k_{y}^{(n)}\right)^{\mathrm{imprim}}\right) & \leq \sum_{\substack{m \mid n \\
m \neq n}} \operatorname{card}\left(X_{y}\left(k_{y}^{(m)}\right)\right) \\
& \leq \sum_{1 \leq m \leq n / 2} C \cdot q_{y}^{m \delta} \\
& =C \cdot \frac{q_{y}^{\left\lfloor\frac{n}{2}\right\rfloor \delta}-1}{1-q_{y}^{-\delta}} \\
& \leq 2 C \cdot q_{y}^{n \delta / 2}
\end{aligned}
$$

as desired.

In the situation of B.1 (b) we also know that $H_{c}^{2 \delta}\left(X_{y} \times{ }_{k_{y}} \bar{k}_{y}, Q_{l}\right)$ has dimension 
1 and the eigenvalue of Frob $_{y}$ is equal to $q_{y}^{\delta}$. Therefore we have

$$
\operatorname{card}\left(X_{y}\left(k_{y}^{(n)}\right)\right) \geq q_{y}^{n \delta}-C \cdot q_{y}^{n\left(\delta-\frac{1}{2}\right)}
$$

Combining this with Lemma B.3 we deduce

$$
\begin{aligned}
\operatorname{card}\left\{x \in|X| \mid f(x)=y \text { and }\left[k_{x} / k_{y}\right]=n\right\} & \geq \frac{1}{n} \cdot\left(\operatorname{card}\left(X_{y}\left(k_{y}^{(n)}\right)\right)-2 C \cdot q_{y}^{n \delta / 2}\right) \\
& \geq \frac{1}{n} \cdot\left(q_{y}^{n \delta}-C \cdot q_{y}^{n\left(\delta-\frac{1}{2}\right)}-2 C \cdot q_{y}^{n \delta / 2}\right) \\
& \geq \frac{1}{n} \cdot\left(q_{y}^{n \delta}-3 C \cdot q_{y}^{n\left(\delta-\frac{1}{2}\right)}\right),
\end{aligned}
$$

as desired.

Now fix an integral scheme $X$ of finite type over Spec $\mathbb{Z}$ and of dimension $d>0$. For any subset $S \subset|X|$ and a complex parameter $s$ we define

$$
F_{S}(s):=\sum_{x \in S} q_{x}^{-s}
$$

\section{Proposition B.5.}

(a) This series converges absolutely and locally uniformly for $\operatorname{Re}(s)>d$. Thus it defines a holomorphic function in this region.

(b) We have

$$
\lim _{s \backslash d} F_{|X|}(s)=\infty
$$

where the limit is taken with $s$ approaching $d$ along the real line from the positive direction.

Proof. Let $K$ be the function field of $X$, and assume first that $\operatorname{char}(K)>0$. Let $\mathbb{F}_{q}$ be its field of constants, of cardinality $q$. Note that for both assertions we may replace $X$ by a Zariski dense open subscheme (using noetherian induction for (a)). Thus we may suppose that $X$ is a geometrically irreducible scheme over $Y:=\operatorname{Spec} \mathbb{F}_{q}$. Then we have

$$
F_{S}(s)=\sum_{n \geq 1} q^{-n s} \cdot \operatorname{card}\left\{x \in S \mid\left[k_{x} / F_{q}\right]=n\right\} .
$$


Taking absolute values and using Proposition B.1 (a) we see that this series is dominated by

$$
\sum_{n \geq 1} q^{-n \operatorname{Re}(s)} \cdot \frac{1}{n} \cdot C \cdot q^{n d}=C \cdot\left|\log \left(1-q^{d-\operatorname{Re}(s)}\right)\right|
$$

Clearly this is locally uniformly bounded for $\operatorname{Re}(s)>d$, proving (a). For (b) we take $s \in \boldsymbol{R}$ and calculate, using Proposition B.1 (b):

$$
\begin{aligned}
F_{|X|}(s) & =\sum_{n \geq 1} q^{-n s} \cdot \operatorname{card}\left\{x \in|X| \mid\left[k_{x} / F_{q}\right]=n\right\} \\
& \geq \sum_{n \geq 1} q^{-n s} \cdot \frac{1}{n} \cdot\left(q^{n d}-C^{\prime} \cdot q^{n\left(d-\frac{1}{2}\right)}\right) \\
& =-\log \left(1-q^{d-s}\right)+C^{\prime} \cdot \log \left(1-q^{d-\frac{1}{2}-s}\right) .
\end{aligned}
$$

This implies assertion (b).

The case $\operatorname{char}(K)=0$ is treated in essentially the same fashion, with $Y=\operatorname{Spec} F_{q}$ replaced by the spectrum of the integral closure of $Z$ in $K$. One first carries out the above calculation for the fibers over all $y \in|Y|$, and then estimates the remaining sum over $y$ as in the number field case.

Definition B.6. If the limit

$$
\mu_{X}(S):=\lim _{s \backslash d} \frac{F_{S}(s)}{F_{|X|}(s)}
$$

exists, we say that $S$ has a (Dirichlet) density and $\mu_{X}(S)$ is called the (Dirichlet) density of $S$ (in $X)$.

The following facts are straightforward to verify.

\section{Proposition B.7.}

(a) If $S$ has a density, then $0 \leq \mu_{X}(S) \leq 1$.

(b) The set $|X|$ has density 1.

(c) If $S$ is contained in a Zariski closed proper subset of $X$, then $S$ has density 0 . 
(d) If $S_{1} \subset S \subset S_{2} \subset|X|$ such that $\mu_{X}\left(S_{1}\right)$ and $\mu_{X}\left(S_{2}\right)$ exist and are equal, then $\mu_{X}(S)$ exists and is equal to $\mu_{X}\left(S_{1}\right)=\mu_{X}\left(S_{2}\right)$.

(e) For any subsets $S_{1}, S_{2} \subset|X|$, if three of the following densities exist, then so does the fourth and we have

$$
\mu_{X}\left(S_{1} \cup S_{2}\right)+\mu_{X}\left(S_{1} \cap S_{2}\right)=\mu_{X}\left(S_{1}\right)+\mu_{X}\left(S_{2}\right)
$$

(f) Let $f: X \rightarrow Y$ be a dominant morphism of integral schemes of finite type over $\operatorname{Spec} Z$. Suppose that $\operatorname{dim}(X)=\operatorname{dim}(Y)$ and that $f$ is totally inseparable at the generic point. Then any given subset $S \subset|X|$ has a density if and only if $f(S)$ has a density, and then $\mu_{X}(S)=\mu_{Y}(f(S))$.

Proof. Assertions (a), (b), (d), and (e) are clear from the definition. The statement (c) follows from Proposition B.5, because we obtain that $F_{S}(s)$ converges near $s=d$ while $F_{|X|}(s)$ diverges. To prove (f) we choose a Zariski dense open subset $V \subset Y$ such that $U:=f^{-1}(V) \rightarrow V$ is finite and totally inseparable at every point. Using assertions (c-e) we may replace $X$ by $U$ and $Y$ by $V$. Then $f$ induces isomorphisms on the residue fields, hence we have $F_{S}(s)=F_{f(S)}(s)$ for any subset $S \subset|X|$. Now the assertion (f) follows immediately.

The following proposition is a generalization of the fact that the set of primes of absolute degree 1 in a number field has Dirichlet density 1 (with respect to that field!).

Proposition B.8. Let $f: X \rightarrow Y$ be a morphism of schemes of finite type over $\mathrm{Spec} Z$. Suppose that $X$ is integral and that $f$ is non-constant. Then the set

$$
\left\{x \in|X| \mid\left[k_{x} / k_{f(x)}\right]=1\right\}
$$

has Dirichlet density 1.

Proof. First we replace $Y$ by the closure of the image of $f$. Next we abbreviate $d:=\operatorname{dim}(X)$ and $e:=\operatorname{dim}(Y)$, and choose a Zariski dense open subset $U \subset X$ such that the fiber dimension of $\left.f\right|_{U}: U \rightarrow Y$ is everywhere equal to $\delta:=d-e$. By Proposition B.7 we may replace $X$ by $U$. Now put

$$
S:=\left\{x \in|X| \mid\left[k_{x} / k_{f(x)}\right] \geq 2\right\} .
$$


By Proposition B.5 (b) it suffices to show that $F_{S}(s)$ converges absolutely and uniformly near $s=d$. Taking absolute values and using Proposition B.1 (a) we see that this series is dominated by

$$
\begin{aligned}
\sum_{x \in S} q_{x}^{-\operatorname{Re}(s)} & =\sum_{y \in|Y|} \sum_{\substack{x \in S \\
f(x)=y}} q_{y}^{-\left[k_{x} / k_{y}\right] \cdot \operatorname{Re}(s)} \\
& =\sum_{y \in|Y|} \sum_{n \geq 2} q_{y}^{-n \operatorname{Re}(s)} \cdot \operatorname{card}\left\{x \in|X| \mid f(x)=y \quad \text { and } \quad\left[k_{x} / k_{y}\right]=n\right\} \\
& \leq \sum_{y \in|Y|} \sum_{n \geq 2} q_{y}^{-n \operatorname{Re}(s) \cdot \frac{1}{n} \cdot C \cdot q_{y}^{n \delta}} \\
& \leq \sum_{y \in|Y|} C \cdot \frac{q_{y}^{2(\delta-\operatorname{Re}(s))}}{1-q_{y}^{\delta-\operatorname{Re}(s)}} \\
& \leq \frac{C}{1-2^{\delta-\operatorname{Re}(s)}} \cdot F_{|Y|}(2(\operatorname{Re}(s)-\delta))
\end{aligned}
$$

By Proposition B.5 (a) this converges locally uniformly for $\operatorname{Re}(s)>\delta+\frac{e}{2}=d-\frac{e}{2}$. Since $f$ is non-constant, we have $e>0$ and hence uniform convergence near $s=d$, as desired.

Now we come to the Čebotarev density theorem. Consider a finite étale Galois covering $\tilde{X} \rightarrow X$ with Galois group $G$ such that $\tilde{X}$ is irreducible. The Frobenius substitution of any point $\tilde{x} \in|\tilde{X}|$ over its image point $x$ is a unique element of $G$. The conjugacy class of this element depends only on $x$ and is denoted Frob .

Theorem B.9. For every conjugacy class $\mathscr{C} \subset G$ the set

$$
\left\{x \in|X| \mid \text { Frob }_{x}=\mathscr{C}\right\}
$$

has Dirichlet density

$$
\frac{\operatorname{card}(\mathscr{C})}{\operatorname{card}(G)}
$$

Proof. From the representation theory of finite groups we know that the vector space of central functions $G \rightarrow C$ has (at least) two natural bases, namely the characteristic functions of the conjugacy classes in $G$, respectively 
the irreducible characters of $G$. Let us denote the latter by $\varphi_{i}$, with $\varphi_{0} \equiv 1$ being the trivial character. If $\varphi_{\mathscr{C}}$ is the characteristic function of a conjugacy class $\mathscr{C}$, we have $\varphi_{\mathscr{C}}=\Sigma_{i} a_{\mathscr{C}, i} \varphi_{i}$ with

$$
a_{\mathscr{C}, 0}=\left(\varphi_{\mathscr{C}}, \varphi_{0}\right)=\frac{\operatorname{card}(\mathscr{C})}{\operatorname{card}(G)} .
$$

Now for any central function $\varphi$ we consider the series

$$
F_{\varphi}(s):=\sum_{x \in|X|} \varphi\left(\operatorname{Frob}_{x}\right) \cdot q_{x}^{-s} .
$$

If $S_{\mathscr{C}}$ denotes the set in the theorem, we clearly have $F_{S_{\mathscr{C}}}(s)=F_{\varphi_{\mathscr{C}}}(s)$. On the other hand we have $F_{|X|}(s)=F_{\varphi_{0}}(s)$. Thus we must prove

$$
\lim _{s \searrow d} \frac{F_{\varphi_{\mathscr{C}}}(s)}{F_{\varphi_{0}}(s)}=\left(\varphi_{\mathscr{C}}, \varphi_{0}\right)
$$

for every conjugacy class $\mathscr{C}$. This is equivalent to the assertion

$$
\lim _{s \searrow d} \frac{F_{\varphi_{\mathrm{t}}}(s)}{F_{\varphi_{0}}(s)}=\left(\varphi_{i}, \varphi_{0}\right)= \begin{cases}1 & \text { if } i=0, \\ 0 & \text { if } i \neq 0,\end{cases}
$$

for all $i$. This is obvious when $i=0$, so by Proposition B.5 (b) it suffices to prove that $F_{\varphi}(s)$ bounded near $s=d$ for any non-trivial irreducible character $\varphi$. In the following we fix such $\varphi$.

As in the proof of Proposition B.5 we first suppose that $X$ is a geometrically irreducible scheme over $Y:=\operatorname{Spec} \mathbb{F}_{q}$. Then we can rewrite

$$
F_{\varphi}(s)=\sum_{n \geq 1} q^{-n s} \cdot \sum_{\substack{x \in|X| \\\left[\boldsymbol{x}_{\boldsymbol{x}} / \mathbb{F}_{q}\right]=n}} \varphi\left(\mathrm{Frob}_{x}\right) .
$$

I claim that this differs from

$$
\sum_{n \geq 1} q^{-n s} \cdot \frac{1}{n} \cdot \sum_{x \in X\left(F_{q} n\right)} \varphi\left(\text { Frob }_{x}\right)
$$

by a function which is bounded near $s=d$. Indeed, since any point $x \in|X|$ with $\left[k_{x} / \mathbb{F}_{q}\right]=n$ corresponds to precisely $n$ primitive points of $X\left(\mathbb{F}_{q^{n}}\right)$, the difference is equal to

$$
\sum_{n \geq 1} q^{-n \operatorname{Re}(s)} \cdot \frac{1}{n} \cdot \sum_{x \in X\left(F_{q}^{n}\right)^{\text {imprim }}} \varphi\left(\text { Frob }_{x}\right)
$$


By Lemma B.3 this is bounded by

$$
\sum_{n \geq 1} q^{-n \operatorname{Re}(s)} \cdot \frac{1}{n} \cdot \operatorname{Const} \cdot q^{n d / 2} \leq \text { Const } \cdot\left|\log \left(1-q^{d / 2-\operatorname{Re}(s)}\right)\right|
$$

As $d \geq 1$, this is indeed bounded near $s=d$.

To evaluate formula (B.10) we use the Lefschetz trace formula in étale cohomology. Choose a number field $E \subset C$ such that the irreducible representation $\rho$ of $G$ with $\varphi=\operatorname{tr} \circ \rho$ can be defined over $E$. Choose a prime $l \nmid q$ and an embedding $E \varsigma \bar{Q}_{l}$. Then $\rho$ gives rise to a representation of the étale fundamental group of $X$ over $\bar{Q}_{l}$ and thus to a lisse $\bar{Q}_{l}$-sheaf $\mathscr{F}_{l}$. By construction we have $\varphi\left(\operatorname{Frob}_{x}\right)=\operatorname{tr}\left(\left.\operatorname{Frob}_{x}\right|_{\mathscr{F}_{l, \bar{x}}}\right)$ for every point $x$ of $X$ over a finite field. The Lefschetz trace formula (see [6] Rapport Th.3.2) thus asserts that

$$
\sum_{x \in X\left(F_{q} n\right)} \varphi\left(\operatorname{Frob}_{x}\right)=\sum_{i=0}^{2 d}(-1)^{i} \operatorname{tr}\left(\left.\operatorname{Frob}_{q}^{n}\right|_{H_{c}^{i}\left(X \times_{F_{q}} \bar{F}_{q}, \mathscr{F}_{l}\right)}\right)
$$

for every $n \geq 1$. Moreover, since $\mathscr{F}_{l}$ is pointwise pure of weight 0 , we know by Deligne [7] Th. 3.3.1 that the eigenvalues of Frob ${ }_{q}$ on $H_{c}^{i}\left(X \times_{F_{q}} \bar{F}_{q}, \mathscr{F}_{l}\right)$ are algebraic numbers of complex absolute value $\leq q^{i / 2}$.

Let us first consider all the terms with $i<2 d$. The corresponding part of (B.10) is bounded by

$$
\sum_{n \geq 1} q^{-n \operatorname{Re}(s)} \cdot \frac{1}{n} \cdot \text { Const } \cdot q^{n\left(d-\frac{1}{2}\right)} \leq \text { Const } \cdot\left|\log \left(1-q^{d-\frac{1}{2}-\operatorname{Re}(s)}\right)\right|
$$

which is clearly bounded near $s=d$. Next let $\boldsymbol{F}_{\tilde{q}}$ denote the constant field of $\tilde{X}$ and $G^{\prime \prime}$ its Galois group over $F_{q}$. We have a natural surjection $G \rightarrow G^{\prime \prime}$ whose kernel comes from the geometric fundamental group of $X$. Now $H_{c}^{2 d}\left(X \times_{F_{q}} \bar{F}_{q}, \mathscr{F}_{l}\right)$ is zero unless $\mathscr{F}_{l}$ is geometrically trivial, i.e. unless $\varphi$ comes from some irreducible character $\varphi^{\prime \prime}$ of $G^{\prime \prime}$. Since $G^{\prime \prime}$ is cyclic, this must be an abelian character of degree 1 . Therefore in that case the dimension of $H_{c}^{2 d}\left(X \times{ }_{F_{q}} \bar{F}_{q}, \mathscr{F}_{l}\right)$ is 1 and the eigenvalue of Frob $_{q}$ is equal to $\varphi^{\prime \prime}\left(\mathrm{Frob}_{q}\right) \cdot q^{d}$. The remaining part of (B.10) is thus equal to

$$
\sum_{n \geq 1} q^{-n s} \cdot \frac{1}{n} \cdot\left(\varphi^{\prime \prime}\left(\text { Frob }_{q}\right) \cdot q^{d}\right)^{n}=-\log \left(1-\varphi^{\prime \prime}\left(\text { Frob }_{q}\right) \cdot q^{d-s}\right)
$$

Here $\varphi^{\prime \prime}\left(\mathrm{Frob}_{q}\right)$ is a root of unity, which is non-trivial since $\varphi$ and hence 
$\varphi^{\prime \prime}$ is a non-trivial character. Thus this term extends to a holo-morphic function near $s=d$, as desired. This finishes the proof of Theorem B.9 in the positive case.

The modifications for characteristic zero are the same as in the proof of Proposition B.5. The main difference occurs at the very end of the proof, where the remaining part of $F_{\varphi}(s)$ comes out to be essentially

$$
\sum_{y \in|Y|} \varphi^{\prime \prime}\left(\text { Frob }_{y}\right) \cdot q_{y}^{d-1-s}
$$

where $\varphi^{\prime \prime}$ is a (not necessarily abelian) Artin character. Estimating this function amounts simply to the usual Čebotarev theorem in the number field case (see e.g. Neukirch [23] Kap.VII Th.13.4), which finishes the proof.

\section{References}

[ 1 ] Anderson, G., t-Motives, Duke Math. J., 53, 2 (1986), 457-502.

[2] Bourbaki, N., Groupes et Algèbres de Lie, chap. 7-8, Paris, Masson (1990).

[ 3 ] Brown, M. L., Singular Moduli and Supersingular Moduli of Drinfeld Modules, Invent. Math., 110 (1992), 419-439.

[ 4 ] Chi, W., $l$-adic and $\lambda$-adic representations associated to abelian varieties defined over number fields, Amer. J. Math., 114 (1992), 315-354.

[ 5 ] David, C., Average Distribution of supersingular Drinfeld Modules, J. Number Theory, 56 (1996), 366-380.

[6] Deligne, P., Cohomologie Etale, LNM 569, Berlin etc., Springer 1977.

[ 7 ] L La Conjecture de Weil, II, Publ. Math. IHES, 52 (1980), 137-252.

[ 8 ] Hodge Cycles on Abelian Varieties, in: Hodge Cycles, Motives, and Shimura Varieties, Deligne, P., et al. (Eds.), ch. I, LNM 900, Berlin etc., Springer (1982), 9-100.

[ 9 ] Deligne, P. and Husemoller, D., Survey of Drinfeld Modules, Contemp. Math., 67 (1987), 25-91.

[10] Drinfeld, V. G., Elliptic Modules (Russian), Math. Sbornik, 94 (1974), 594-627, = Math. USSR-Sb., 23 (1974), 561-592.

[11] Elliptic Modules II (Russian), Math. Sbornik, 102 (1977), 182-194, = Math. USSR-Sb., 31 (1977), 159-170.

[12] Dynkin, E.B., Semisimple Subalgebras of Semisimple Lie Algebras, Am. Math. Soc. Transl. Ser. 2, 6 (1957), 111-244.

[13] Fried, M. D. and Jarden, M., Field Arithmetic, Berlin etc., Springer, 1986.

[14] Gekeler, E.-U., De Rham Cohomology for Drinfeld Modules, Sém. Théorie des Nombres, Paris 1988-89, Boston etc., Birkhäuser (1990), 57-85.

[15] Goss, D., $L$-series of $t$-motives and Drinfeld Modules, in: The Arithmetic of Function Fields, Goss, D., et al. (Eds.) Proceedings of the workshop at Ohio State University 1991. Berlin, Walter de Gruyter (1992), 313-402.

[16] - Drinfeld Modules: Cohomology and Special Functions, in: Motives, Jannsen, U., et al (Eds.), Proceedings of the summer research conference on motives, Seattle 1991, Proc. Symp. Pure Math., 55 Part 2 (1994), 309-362.

[17] - Basic Structures of Function Field Arithmetic, Ergebnisse 35, Berlin etc., Springer, 
1996.

[18] Hayes, D. R., Explicit Class Field Theory in Global Function Fields, in: Studies in Algebra and Number Theory, Adv. Math., Suppl. Stud. 6, Academic Press (1979), 173-217.

[19] - A Brief Introduction to Drinfeld Modules, in: The Arithmetic of Function Fields, Goss, D.,et al. (Eds.) Proceedings of the workshop at Ohio State University 1991. Berlin, Walter de Gruyter (1992), 1-32.

[20] Humphreys, J.E., Linear Algebraic Groups, GTM 21, New York etc, Springer (1975), (1981)

[21] Jouanolou, J.-P., Théorèmes de Bertini et Applications, Progress in Math. 42, Boston etc., Birkhäuser, 1983.

[22] Katz, N.M., Gauß Sums, Kloosterman Sums, and Monodromy Groups, Ann. of Math. Stud. 116, Princeton: Princeton Univ. Press., 1988.

[23] Neukirch, J., Algebraische Zahlentheorie, Berlin etc., Springer, 1992.

[24] Ogus, A., Hodge Cycles and Crystalline Cohomology, in: Hodge Cycles, Motives, and Shimura Varieties, Deligne, P., et al. (Eds.), ch. VI, LNM 900, Berlin etc., Springer (1982), 357-414.

[25] Pink, R., Compact Subgroups of Linear Algebraic Groups, Preprint, August 1996.

[26] Serre, J.-P., Abelian l-adic Representations and Elliptic Curves, New York, W. A. Benjamin, 1968.

[27] Groupes Algébriques Associés aux Modules de Hodge-Tate, Astérisque 65 (1979), 155-188.

[28] — Letter to J. Tate, Jan. 2, 1985.

[29] - Résumés des cours au Collége de France, Annuaire du Collège de France (1984-85), 85-90.

[30] - Résumés des cours au Collège de France, Annuaire du Collège de France (1985-86), 95-99.

[31] Taguchi, Y., Semi-simplicity of the Galois Representations Attached to Drinfeld Modules over Fields of "Infinite Characteristics", J. Number Theory, 44 (1993), 292-314.

[32] - The Tate Conjecture for $t$-Motives, Proc. Am. Math. Soc., 123 (1995), 3285-3287.

[33] Tamagawa, A., The Tate Conjecture for A-Premotives, Preprint 1994. 
